\title{
Energy-driven disorder in mean field QCD
}

\author{
Sergei Nedelko $\odot^{*}$ and Vladimir Voronin $\odot^{\dagger}$ \\ Bogoliubov Laboratory of Theoretical Physics, JINR, 141980 Dubna, Russia
}

(Received 16 December 2020; accepted 1 June 2021; published 22 June 2021)

\begin{abstract}
The impact of the finite-size effects on the vacuum free energy density of full QCD with $N_{f}$ massless flavors in the presence of a homogeneous (anti-)self-dual Abelian background gluon field is studied. The zero-temperature free energy density of the four-dimensional spherical domain is computed as a function of the background field strength $B$ and domain radius $R$. The calculation is performed in the one-loop approximation improved by accounting for mixing of the quark and gluon quasizero modes with normal modes, with the use of the $\zeta$-function regularization. It is indicated that, under plausible assumptions on the character of the mixing, the quantum correction to the free energy density has a minimum as a function of $B$ and $R$. Within the mean-field approach to the QCD vacuum based on the domain wall network representation of the mean field, the existence of the minimum may prevent infinite growth of individual domains, thus protecting the vacuum from the long-range ordering, and hence serving as the origin of disorder in the statistical ensemble of domain wall networks, driven by the minimization of the overall free energy of the dominant gauge field configurations.
\end{abstract}

DOI: 10.1103/PhysRevD.103.114021

\section{INTRODUCTION}

It is generally accepted that the physical QCD vacuum can be characterized by various gluon, quark, and mixed condensates. The condensates have played an important role in understanding the basic features of hadron physics. In particular, the lowest-dimension condensates $\left\langle g^{2} F^{2}\right\rangle$, $\left\langle\left(g^{2} \tilde{F} F\right)^{2}\right\rangle$, and $\langle\bar{\psi} \psi\rangle$ are relevant to the anomalous breakdown of scale and $U_{\mathrm{A}}(1)$ symmetries, and the spontaneous breaking of chiral $S U_{\mathrm{L}}\left(N_{f}\right) \times S U_{\mathrm{R}}\left(N_{f}\right)$ symmetry.

In principle, the condensates could be described in terms of the background (vacuum) gluon fields within the self-consistent mean-field approach to the QCD vacuum. However, the necessity to express the condensateswhich are vacuum expectation values of the color-neutral composite fields-in terms of the vector potential of the background gauge field combined with the strong-coupling regime complicates the actualization of the mean-field approach to the QCD vacuum.

Depending on the assumptions about the structure of the vacuum, the gauge mean-field configurations underlying the condensates have been taken in various forms, ranging from a superposition of quasiclassical gluon

\footnotetext{
*nedelko@theor.jinr.ru

voronin@theor.jinr.ru
}

Published by the American Physical Society under the terms of the Creative Commons Attribution 4.0 International license. Further distribution of this work must maintain attribution to the author(s) and the published article's title, journal citation, and DOI. Funded by SCOAP. configurations like the instanton gas or liquid [1,2] to the fields with a constant field strength squared, $g^{2} F^{2}=$ const, representing the global minimum of the quantum effective action of QCD. Properties of the quantum effective action for homogeneous gluon fields were studied in various approaches [3-13]. In particular, Leutwyler has demonstrated that the covariantly constant Abelian (anti-)self-dual field

$$
\begin{aligned}
\hat{B}_{\mu} & =-\frac{1}{2} \hat{n} B_{\mu \nu} x_{\nu}, \quad \tilde{B}_{\mu \nu}= \pm B_{\mu \nu} B_{\mu \rho}=B^{2} \delta_{\nu \rho}, \\
\hat{n} & =t_{3} \cos \xi+t_{8} \sin \xi
\end{aligned}
$$

is singled out from other gluon fields as the only gaugefield configuration with constant strength that is stable against small gluon and quark fluctuations and, hence, could be considered as a likely contender for the global minimum of the effective action [8]. The stability was understood as the absence of the tachyonic modes in the spectrum of small quantum fluctuations in a given background gluon field. An obvious shortcoming of the purely homogeneous gauge field as a candidate for the mean vacuum field is that it would describe a globally ordered vacuum state and thus break all of the symmetries of QCD. However, this argument does not apply to the more complicated relevant case when the gluon fields minimizing the quantum effective action-the vacuum fields-are arranged in domains of a homogeneous Abelian (anti-)selfdual gluon field; the sizes and shapes of the domains in $R^{4}$ randomly vary around certain mean values [14]. As a 
whole, such a set can be characterized as the statistical ensemble of gauge fields that are homogeneous Abelian (anti-)self-dual almost everywhere in $R^{4}$ besides the boundaries between domains where the field appears to be neither homogeneous nor Abelian (anti-)self-dual. It has to be noted that the topological charge density distribution in the typical gauge-field configurations was studied within lattice QCD with dynamical quarks, with the results supporting the picture of entangled space-time regions of sign-alternating topological charge [15-17].

An instance of disordered lumpy configurations is seen within the Ginzburg-Landau (GL) model of the quantum effective action of QCD [14,18,19]. In this approach, an almost-everywhere homogeneous Abelian (anti-)self-dual gluon field can be represented by the domain wall networks, arising straightforwardly as soon as the existence of the nonzero scalar gluon condensate $\left\langle g^{2} F^{2}\right\rangle$ is assumed. Domain wall networks come out of the structure of the degenerate discrete global minima of the GL effective potential, related to each other via discrete symmetry transformations, i.e., $C P$ and Weyl reflections in the root space of the color $s u(3)$ algebra.

The condensates are the key notion of the Wilson operator product expansion formalism applied to the phenomenology of QCD and hadron physics. However, there are potential inconsistencies in the definition of condensates attributed to infrared renormalons (see, e.g., Refs. [20,21] and references therein for discussion and technical details). The major point is that operator product expansion coefficients are not determined exclusively by perturbation theory, and the infrared behavior of correlation functions should be considered properly [22] which can be achieved by explicit separation of infrared and ultraviolet regions at the normalization scale $\mu$. The region with momenta larger than $\mu$ is treated perturbatively. Without this explicit separation, the pole of the free gluon propagator at zero momentum would lead to infrared renormalons in QCD. In contrast, explicit propagators in the background field (1) are regular at zero momentum but still reproduce an ultraviolet regime of free propagators. The simplest example is the scalar field propagator in a homogeneous Abelian (anti-)self-dual gluon field [7],

$$
\frac{1-\exp \left(-p^{2} / B\right)}{p^{2}},
$$

which manifestly demonstrates the character of modifications at low momenta. Moreover, the above propagator is an entirely analytical function in the complex momentum plane. The quark propagator is analytical as well, which can be interpreted as the confinement of dynamical quarks in the presence of an Abelian (anti-)self-dual gauge field.

Almost-everywhere homogeneous Abelian (anti-)selfdual gluon fields have been incorporated into the hadronization scheme within the domain model of the QCD vacuum [23-28]. The Abelian (anti-)self-dual nature ensures the confinement of both dynamical and static quarks (absence of poles in the propagators of color-charged fields as well as fulfillment of the area law for the Wilson loop), the resolution of the $U_{A}(1)$ problem, and the spontaneous breakdown of chiral symmetry. With a minimal set of parameters, the domain model provided a universal and rather accurate description of the masses and various decay constants of light and heavy-light mesons and heavy quarkonia, including their excited states, as well as some form factors. The picture of the QCD vacuum based on the Abelian (anti-)self-dual mean field turned out to be convenient for exposing a catalyzing impact of a strong electromagnetic field on quark deconfinement [14,18,29-31].

On the whole, these rather satisfactory phenomenological applications inspire the task of clearing up the mechanism behind the balance between the competitive tendencies for a long-range order in the ground state and disorder that may originate from two complementary origins: the topologically stable defects in the background field and the existence of a minimum of the effective action with respect to the size of the regions of homogeneity (domain size). Topologically nontrivial gluon field configurations are expected to emerge through the division of the arbitrary gauge field into Abelian and non-Abelian parts, and may be seen in the domain wall network as frustrations of the color and space-time orientation of the background field at the domain wall junctions. We shortly comment on this mechanism below but do not discuss it in detail here. A discussion of relevant physics can be found in the review [32] and references therein.

The existence of a minimum of the effective action with respect to the domain size can be called the energy-driven origin of disorder, which is the main subject of the present paper. The quark and gluon quasizero modes characteristic of the covariantly constant Abelian (anti-)self-dual background field in a finite region may play a peculiar role in the formation of domains. An infinite number of quasizero modes become degenerate zero modes in the limit of infinite domain size, and thus potentially lead to an infrared singular behavior of the effective potential. In the plain oneloop approximation, the contributions of the quark and gluon quasizero modes to the effective potential have opposite signs, and their strong concurrent impact on the potentially IR singular behavior of the effective potential becomes manifest in the infinite-volume limit.

In the present paper, the effective potential for the Abelian (anti-)self-dual field (1) in a four-dimensional spherical domain with radius $R$ is calculated for $S U(3)$ chromodynamics with $N_{f}$ massless quarks using the zeta function regularization, which completes the previously reported results for pure gluodynamics [33]. The quark and gluon fields are subject to the bag-like boundary conditions $[14,25]$. It is important that the interaction of gluon and quark quasizero modes (zero modes in the limit $R \rightarrow \infty$ ) 
with the normal modes is treated beyond one loop, as has been put forward by Leutwyler [8]. This interaction leads to the contribution of the quasizero modes to the effective potential being regular in the limit of infinite domain size. This is crucial for the overall existence of the thermodynamic limit and, as underlined in Ref. [8], the consistency of the strong-field limit of the effective action with asymptotic freedom. In fact, a mixing of quasizero and normal modes has to be taken into account in all-loop orders as there is no good small parameter, which presents a barely solvable task, especially in the finite region. However, as it was demonstrated in Ref. [8], the most significant consequence of the mixing is the emergence of the effective "mass" for the quasizero modes. This observation enables sensible modeling of the plausible form of the dependence of the "effective mass" on the domain size $R$, and the identification of conditions for the existence of the minimum of the effective potential with respect to the radius of the domain $R$ and field strength $B$ inside the domain.

It is shown that formation of the domains with finite size $R$ can be energetically preferable if the effective "mass" falls from asymptotic nonzero value at $R \rightarrow \infty$, fixed by the asymptotic freedom and correct strong field limit, to zero at $R \rightarrow 0$ as it follows from the dependence of normal modes on the domain size. The final result for the free energy density of full QCD is shown in the last figure in Sec. III.C. The minimum in field strength and domain size is clearly present for a wide class of functional dependence of the zero mode effective "mass" on the domain size.

The finite mean size of the domains in the network is determined by the minimum of the effective action density inside individual domains. The existence of the mean domain size that minimizes the action density can be seen as a condition for the sustainability of a domain wall network. In general, there is an infinite set of networks with degenerate values of the quantum effective action that constitutes a statistical ensemble of dominant vacuum gluon field configurations. In accordance with the character of the free energy dependence on domain size, the degree of disorder in the ensemble may vary from a highly disordered distribution of entangled domains with variable size and shape, to the almost periodic distribution of identically sized and shaped domains, reminiscent of spin liquid and (anti)ferromagnetic states, respectively.

In the next section we update the formal framework underlying the domain wall network representation of the background gluon fields in order to adjust it to the formulation of the particular problem studied in this paper. The ghost, gluon, and quark contributions to the free energy density of a spherical domain with Abelian selfdual gauge field are discussed in detail in the third section. The Appendices contain quite involved technical details of calculations. The main difficulty is combination of finite size and background field, and their simultaneous treatment is the result by itself.

\section{EFFECTIVE ACTION OF QCD AND THE DOMAIN WALL NETWORKS}

The initial, gauge-unfixed, Euclidean functional integral representation for the QCD partition function

$$
Z\left[B_{\mathrm{vac}}\right]=N \int_{\mathcal{F}} D A \int_{\Psi} D \bar{\psi} D \psi \exp \{-S[A, \bar{\psi}, \psi]\}
$$

assumes a certain choice of the functional spaces of integration over gluon and quark fields. If one allows nonzero gluon condensates, then the functional space $\mathcal{F}$ has to be subjected to an appropriate condition, such as

$$
\mathcal{F}=\left\{A: \lim _{V \rightarrow \infty} \frac{1}{V} \int_{V} d^{4} x g^{2} F_{\mu \nu}^{a}(x) F_{\mu \nu}^{a}(x)=B_{\text {vac }}^{2}\right\} .
$$

This definition is reminiscent of the Schrödinger functional representation (see, for instance, Refs. [8,34,35]). The difference is that the condition (2) is imposed on the gauge-invariant combination of the gauge fields and has an integral (functional) form.

In order to define the above partition function in the mean-field approach, one has to identify field configurations $\mathcal{B}$ that are potentially relevant to the description of the QCD vacuum with nonzero condensates. Instanton liquid configurations, a center-vortex ensemble, gauge fields with constant field strength, or domain wall networks are all examples of $\mathcal{B}$.

The division of the general gauge fields $A_{\mu}^{a} \in \mathcal{F}$ into the given background fields $B_{\mu}^{a} \in \mathcal{B}$ with an extensive classical action specified by Eq. (2) and the fluctuations $Q_{\mu}^{a}$ in this sample background $B_{\mu}^{a}$ supplemented by the background gauge condition $D(B) Q=0$ lead to the representation [36]

$$
\begin{aligned}
Z\left[B_{\mathrm{vac}}\right]= & N^{\prime} \int_{\mathcal{B}} D B \exp \left\{-S_{\mathrm{eff}}^{V}[B]\right\} \\
= & N^{\prime \prime} \int_{\mathcal{B}} D B \int_{\mathcal{Q}} D Q \\
& \times \int_{\Psi} D \bar{\psi} D \psi \operatorname{det}[D(B) D(B+Q)] \delta[D(B) Q] \\
& \times \exp \left\{-S_{V}[B+Q, \bar{\psi}, \psi]\right\} .
\end{aligned}
$$

At this step, the functional spaces $\mathcal{Q}$ and $\Psi$ are restricted by the condition

$$
\lim _{V \rightarrow \infty}\left(S_{V}[B+Q, \bar{\psi}, \psi]-S_{V}[B]\right)<\infty,
$$

which excludes long-range fields from the set $\mathcal{Q}$ of fluctuations. The integral over the quark $\psi$ and gluon $Q$ fluctuations defines the effective action $S_{\text {eff }}^{V}[B]$ for a given background field $B$. Whether the constant $B_{\text {vac }}$ is nonzero has to be determined by the minima of the quantum 
effective action. Due to the dimensional transmutation in gauge theories, the condensates have to be expressed in terms of the internal scale $\Lambda_{\mathrm{QCD}}$, that is, $B_{\mathrm{vac}} \propto \Lambda_{\mathrm{QCD}}^{2}$. The dimensional transmutation in gauge theories and its connection to gauge field condensates was discussed in Ref. [37]. In the infinite-volume limit $V \rightarrow \infty$, the global minima of $S_{\text {eff }}^{V}[B]$ dominate the integral over background fields $B$ and thus determine the specific class of gluon field configurations relevant to the self-consistent mean-field description of the QCD vacuum.

The properties of the effective action are crucial for the practical implementation of the described scheme. Detailed discussions of the very existence of a nontrivial minimum of the quantum effective action for homogeneous fields can be found in Refs. [3-12]. Some basic properties have been estimated and can be used for the identification of the likely features of the mean field.

Fields with a constant field strength have to be verified for the role of the vacuum mean field $B_{\mu}^{a}$ first. Such a verification has been going on since the late 1970s, when a chromomagnetic covariantly constant Abelian gauge field was suggested for the role of the QCD vacuum [4]. As has already been mentioned, a covariantly constant Abelian (anti-)self-dual gauge field has appeared to be more preferable in many respects, in particular due to the direct relation to the confinement of dynamical colorcharged fields, and chiral symmetry breaking seen also in terms of meson properties through hadronization.

It should be stressed that the condition (2) in no way restricts the background field functional space $\mathcal{B}$ to configurations with constant strength. The representation (3) assumes the division of the arbitrary gauge field $A$ into two parts: the background $B$ and fluctuation $Q$ fields, with simultaneous gauge fixing for the fluctuation part. If one is going to study the Abelian background $B$, then a specific parametrization of the gauge field has to be implemented [32,38-42]. Both the color and space orientations of the background field may appear to be frustrated at some space-time locations, thus making manifest topological singularities in the vector potential which, in general, cover the whole range of defects of various dimensions: domain wall, vortex, monopole, and zero-dimensional instanton-like defects.

More importantly, as has been stressed, in particular according to Faddeev, quantum equations

$$
\frac{\delta S_{\mathrm{eff}}^{V}[B]}{\delta B}=0
$$

"could have soliton solutions, which are absent in the classical limit. In particular, it is not completely crazy idea that quantum Yang-Mills equations have soliton-like solutions due to the dimensional transmutation" [43].

In general, fields that dominate the functional integral in the infinite-volume (thermodynamic) limit belong to the functional subspace $\tilde{\mathcal{B}} \subset \mathcal{B}$. Given this, in the infinitevolume limit one may reduce the integration over background fields $B$ in Eq. (3) to the subspace of dominant (vacuum) fields and arrive at the mean-field representation of the QCD partition function,

$$
\begin{aligned}
Z\left[B_{\mathrm{vac}}\right]= & N^{\prime \prime} \lim _{V \rightarrow \infty} \int_{\tilde{\mathcal{B}}} D \sigma_{B} \int_{\mathcal{Q}} D Q \\
& \times \int_{\Psi} D \bar{\psi} D \psi \operatorname{det}[D(B) D(B+Q)] \delta[D(B) Q] \\
& \times \exp \left\{-S_{V}[B+Q, \bar{\psi}, \psi]\right\},
\end{aligned}
$$

where $D \sigma_{B}$ is a measure of the integration over the space of vacuum gauge fields. A treatment of these possibly solitonlike vacuum fields $B \in \tilde{\mathcal{B}}$ in the functional integral (3) must be nonperturbative, while a perturbative expansion over fluctuations is likely to be applicable for the calculation of various physical quantities [27,28]. It has to be noted that according to the condition (3) the normalization constant $N^{\prime \prime}$ should contain a factor that cancels a trivial extensive contribution of the background field to the classical action $S[B]$ in the infinite-volume limit. Certain prescriptions for the regularization and renormalization of UV divergences are assumed.

The Ginzburg-Landau approach to the quantum effective action of QCD with the effective Lagrangian [14,18,19,25]

$$
\begin{aligned}
& \mathcal{L}_{\text {eff }}=-\frac{1}{4 \Lambda^{2}}\left(D_{\nu}^{a b} F_{\rho \mu}^{b} D_{\nu}^{a c} F_{\rho \mu}^{c}+D_{\mu}^{a b} F_{\mu \nu}^{b} D_{\rho}^{a c} F_{\rho \nu}^{c}\right)-U_{\text {eff }}, \\
& U_{\text {eff }}=\frac{\Lambda^{4}}{12} \operatorname{Tr}\left(C_{1} \breve{f}^{2}+\frac{4}{3} C_{2} \breve{f}^{4}-\frac{16}{9} C_{3} \breve{f}^{6}\right)
\end{aligned}
$$

indicated an intrinsic possibility for a disordered ground state of QCD. Here, $\Lambda$ is a scale, $F_{\mu \nu}^{a}$ is the standard strength tensor for the $S U_{\mathrm{c}}(3)$ color gauge field, $\breve{f}_{\mu \nu}=T^{a} F_{\mu \nu}^{a} / \Lambda^{2}$, and $D_{\mu}^{a b}=\delta^{a b} \partial_{\mu}-i \breve{A}_{\mu}^{a b}$. The effective Lagrangian respects all symmetries of QCD besides scale invariance, and the real constants $C_{i}$ have to be positive to provide a minimum of the effective potential at nonzero gauge field. Given this, one can check that there is a discrete set of global minima corresponding to the covariantly constant Abelian (anti-)self-dual fields,

$$
\begin{gathered}
\breve{A}_{\mu}^{k}=-\frac{1}{2} \breve{n}_{k} F_{\mu \nu} x_{\nu}, \quad \tilde{F}_{\mu \nu}= \pm F_{\mu \nu}, \\
F_{\mu \nu} F_{\mu \nu}=b^{2} \Lambda^{4}, \quad b_{\text {vac }}^{2}=\frac{-C_{2}+\sqrt{C_{2}^{2}+3 C_{1} C_{3}}}{3 C_{3}},
\end{gathered}
$$

where the matrix $\breve{n}_{k}$ belongs to the Cartan subalgebra of $s u(3)$, 
$\breve{n}_{k}=T^{3} \cos \left(\xi_{k}\right)+T^{8} \sin \left(\xi_{k}\right), \quad \xi_{k}=\frac{2 k+1}{6} \pi, k=0,1, \ldots, 5 . \quad \mathcal{L}_{\text {eff }}=-\frac{1}{2} \Lambda^{2} b_{\text {vac }}^{2} \partial_{\mu} \omega \partial_{\mu} \omega-b_{\text {vac }}^{4} \Lambda^{4}\left(C_{2}+3 C_{3} b_{\text {vac }}^{2}\right) \sin ^{2} \omega$.

These minima are connected with each other by discrete parity and Weyl symmetry transformations.

One concludes that the domain wall solutions of the quantum equations of motion emerge as soon as the effective action has a global minimum corresponding to nonzero gluon condensate $\left\langle g^{2} F^{2}\right\rangle$. For instance, if all parameters of the field besides the angle $\omega$ between chromoelectric and chromomagnetic fields are set to the vacuum values, then the initial GL Lagrangian describes a sine-Gordon field $\omega$,

The available standard kink solution describes a planar domain wall between the regions with homogeneous Abelian self-dual and anti-self-dual gluon fields. Topological charge density vanishes on the wall where the chromomagnetic and chromoelectric fields are orthogonal to each other.

More general domain wall configuration emerges if $\omega, \xi$ and $b$ varying simultaneously. In this case, the equations of motion read

$$
\begin{aligned}
-6 b^{\prime} \omega^{\prime}+b^{3} \sin 2 \omega\left(C_{3} b^{2}(\cos 6 \xi+10)+3 C_{2}\right)-3 b \omega^{\prime \prime} & =0, \\
-15 b^{\prime \prime}+3 b\left(-4 C_{1}+2 \omega^{\prime 2}+5 \xi^{\prime 2}\right)-12 C_{2} b^{3}(\cos 2 \omega-3)-2 C_{3} b^{5}(3 \cos 2 \omega-5)(\cos 6 \xi+10) & =0, \\
2 C_{3} b^{6}(3 \cos 2 \omega-5) \sin 6 \xi-15 b\left(2 b^{\prime} \xi^{\prime}+b \xi^{\prime \prime}\right) & =0 .
\end{aligned}
$$

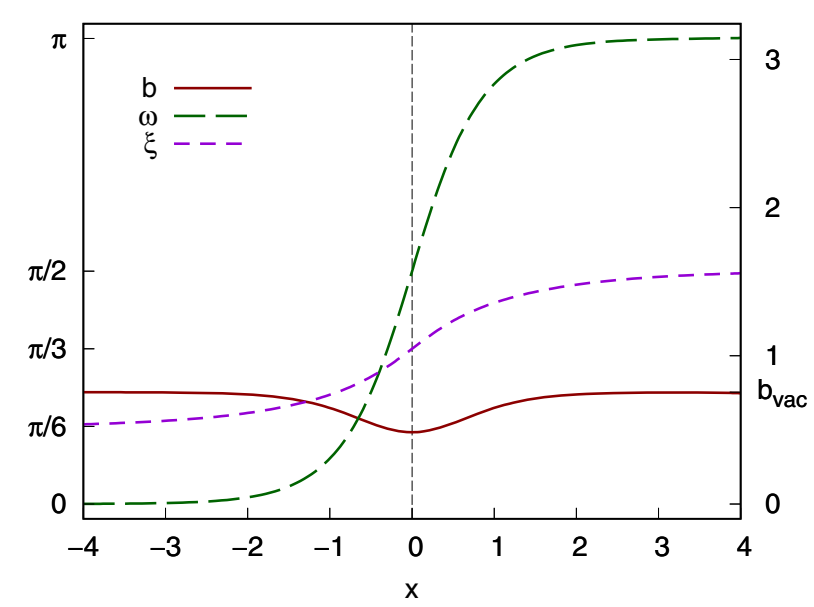

FIG. 1. Domain wall solution of Eq. (8): the gauge field interpolates between two vacuum states, namely, self-dual and anti-self-dual configurations, $\xi$ goes from one boundary of the Weyl chamber to another, and the field strength $b$ has a dip on the wall.
The plain domain wall solution of these equations is shown in Fig. 1. It corresponds to a gauge field that interpolates between two different vacuum configurations. In general, the initial GL Lagrangian may produce more nontrivial soliton-like solutions in both Euclidean and Minkowski spaces. A combination of the additive and multiplicative superpositions of the domain walls allows one to generate various domain walls and domain wall networks in $R^{4}$ [14,44], like the samples shown in Fig. 2.

The Lagrangian (6) has the simplest form, but its symmetry properties and the emergence of the periodic discrete minima as a consequence of the scale invariance breakdown seem to be a general property, qualitatively insensitive to the detailed form of the effective potential. Another form of the strong-field behavior of the GL effective Lagrangian can affect the particulars of the kink solution, but can hardly influence its very existence and general properties. It has to be noted that the role of Weyl reflections in the topology of the QCD ground state has
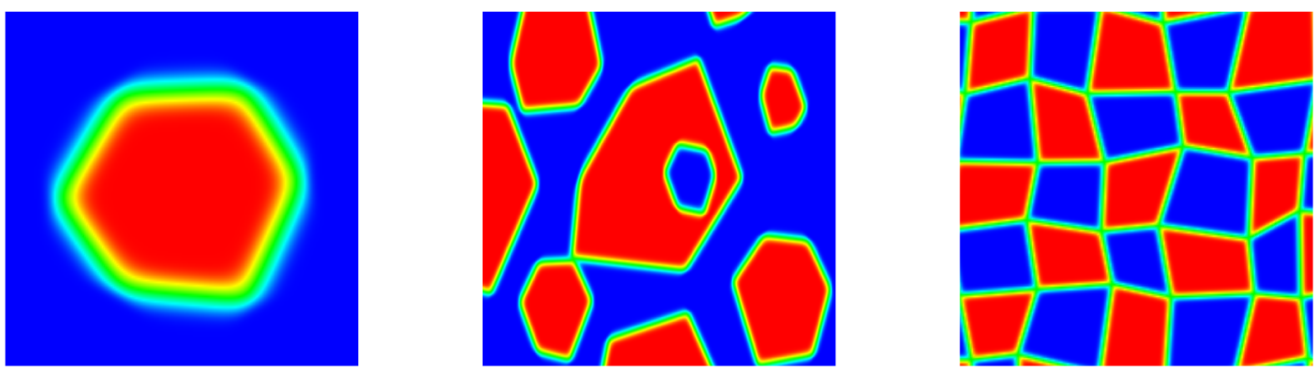

FIG. 2. Two-dimensional slices of topological charge density for various superpositions of domain wall solutions for Eq. (8). Red and blue correspond to Abelian self-dual and anti-self-dual fields. 
been intensively discussed in recent years in the context of the dual superconductor picture of confinement $[32,45]$.

The functional space $\tilde{\mathcal{B}}$ in the integral (5) is assumed to include infinitely many networks with equal values of the free energy. Though an implicit implementation of this prescription within the domain model of the QCD vacuum demonstrated high phenomenological performance $[27,28]$, the conceptual problem of the network stability remains: since domain wall configurations in four-dimensional Euclidean space are not topologically protected and the presence of a kink configuration usually increases the action, any network should evolve to a single infinitely large domain. In the next section, we study a particular effect which may prevent the infinite growth of a single domain.

\section{FREE ENERGY DENSITY}

The Lagrangian (6) does not account for possible finitesize effects. Available evaluations of the effective potential for a homogeneous gauge field were performed in an infinite space-time [3-12] and thus, as a matter of fact, implicitly assumed that the free energy density in the region with a homogeneous field does not depend on the size of the region. Meanwhile, finite-size effects are not excluded and may prevent infinite growth of an individual domain, thus protecting the overall stability of the domain wall network configurations.

The present section is devoted to the study of the dependence of the renormalized free energy density $F(B, R)$ of a finite spherical domain of the Abelian (anti-)self-dual homogeneous field on its radius $R$ and the strength $B$ of the field in full QCD with massless quarks, defined by the finite-volume partition function

$$
\begin{aligned}
\exp ( & \left.-V_{R} F(B, R)\right) \\
= & \operatorname{Ren} N \int_{\mathcal{Q}} D Q \int_{\Psi} D \bar{\psi} D \psi \\
& \times \int_{\mathcal{C}} D \bar{c} D c \exp \left\{-S_{V_{R}}[Q ; \bar{\psi}, \psi ; \bar{c}, c ; B]\right\},
\end{aligned}
$$

where $V_{R}$ is the volume of a four-dimensional ball with radius $R$, which is an idealization of the domain shown in the leftmost picture in Fig. 2, and $S_{V_{R}}$ is the gauge-fixed action of QCD in the presence of the background gluon field defined by Eq. (1). The Feynman background gauge is used below. The normalization constant $N$ is fixed by the condition

$$
F(0, R)=0 .
$$

The renormalization prescription is specified below. The functional spaces $\mathcal{Q}, \Psi$, and $\mathcal{C}$ contain the quark, gauge, and ghost fields subject to the bag-like boundary conditions

$$
\begin{gathered}
\left.\left(i n e^{i \alpha \gamma_{5}}-1\right) \psi(x)\right|_{x \in \partial V_{R}}=0, \\
\left.\breve{n} Q_{\mu}(x)\right|_{x \in \partial V_{R}}=0, \\
\left.\breve{n} c(x)\right|_{x \in \partial V_{R}}=0 .
\end{gathered}
$$

These boundary conditions were discussed in Refs. [14,25]. The choice assumes that there is a physical boundary of the spherical domain, given by the domain wall illustrated in Fig. 1. Bag-like boundary conditions are required by the qualitatively different character of field fluctuations in the bulk of the domain (confining self-dual background field) and on the boundary (chromomagnetic field) [14].

The functional integral (9) is defined through the decomposition of the quark, gauge, and ghost fields,

$$
\begin{aligned}
Q(x) & =\sum_{n} q_{n} Q^{(n)}(x), \quad c(x)=\sum_{n} c_{n} C_{n}(x), \\
\psi(x) & =\sum_{n} \theta_{n} \Psi_{n}(x),
\end{aligned}
$$

over an orthogonal normalized complete set of functions in $\Psi, \mathcal{Q}$, and $\mathcal{C}$. It is convenient to diagonalize the quadratic part of the action, using the eigenfunctions of the corresponding differential operators,

$$
\begin{gathered}
\hat{\not} \Psi_{n}=\lambda_{n}^{\mathrm{q}} \Psi_{n}, \\
-\breve{D}^{2} C_{n}=\lambda_{n}^{\mathrm{gh}} C_{n}, \\
{\left[-\breve{D}^{2} \delta_{\mu \nu}+2 i \breve{B}_{\mu \nu}\right] Q_{\nu}^{(n)}=\lambda_{n}^{\mathrm{gl}} Q_{\mu}^{(n)},}
\end{gathered}
$$

subject to the boundary conditions (11), (12), and (13). The index $n$ denotes all relevant quantum numbers as described below. The quark, gluon, and ghost spectra are purely discrete for any $R$ if the field strength $B$ is nonzero. At finite $R$, all eigenvalues are nonzero for quark fields, and positive for gauge and ghost fields. The one-loop correction $\delta U$ to the classical action

$$
U_{\mathrm{cl}}=\int_{V_{R}} d^{4} x \frac{1}{4 g^{2}}\left(B_{\mu \nu}^{a}\right)^{2}=\frac{\pi^{2} B^{2} R^{4}}{2 g^{2}}
$$

is given by the determinants

$$
\begin{aligned}
\exp (-\delta U)= & N\left[\operatorname{det}\left(-\breve{D}^{2} \delta_{\mu \nu}+2 i \breve{B}_{\mu \nu}\right)\right]^{-\frac{1}{2}} \\
& \times \operatorname{det}\left(-\breve{D}^{2}\right)[\operatorname{det} i \not D]^{N_{f}},
\end{aligned}
$$

where $N_{f}$ is the number of massless quark flavors. Renormalized functional determinants are calculated below by means of analytical regularization, 


$$
\operatorname{Tr} \log \Delta=-\left.\frac{d}{d s} \sum_{n} \lambda_{n}^{-s}\right|_{s=0}=-\left.\frac{d}{d s} \zeta(s)\right|_{s=0},
$$

where $\lambda_{n}$ are eigenvalues of the operator $\Delta$. The computation of $\zeta(s)$ is based on the method summarized in Refs. [46,47]. The contributions of ghosts and gluons were calculated in Ref. [33], and we provide them below for completeness.

\section{A. Ghost contribution to the free energy density}

The evaluation of the ghost contribution to the free energy density is rather straightforward. Details of the solution of the eigenvalue problem [Eqs. (15) and (13)], are given in Ref. [25]. Ghost eigenfunctions are expressed in terms of the confluent hypergeometric function $M(a, b, z)$. The eigenvalues are defined by Eq. (15), which can be written in the form

$$
\begin{aligned}
& M\left(\frac{k}{2}+1-m-\frac{\lambda^{2}}{2 v_{a} B}, \quad k+2, \frac{v_{a} B R^{2}}{2}\right)=0, \\
& k=0,1,2, \ldots, \quad m=-\frac{k}{2}, \quad-\frac{k}{2}+1, \ldots, \frac{k}{2},
\end{aligned}
$$

where $v_{a}$ is the absolute value of the ath nonzero eigenvalue of the matrix $\breve{n}=n^{a} T^{a}$, and $T^{a}$ are generators in the adjoint representation. The eigenmodes corresponding to the zero eigenvalues of $\breve{n}$ do not contribute to the free energy density $F(B, R)$ due to the normalization condition (10). Eigenvalues with a given $k, m$, radial number $r$, and color index $a$ are $(k+1)$ degenerate. The ghost contribution takes the form

$$
\delta U^{\mathrm{gh}}=-\sum_{k m r} \operatorname{Tr} \ln \frac{\lambda_{k m r}^{2}(v B, R)}{\lambda_{k m r}^{2}(0, R)}=\left.\frac{d}{d s} \zeta^{\mathrm{gh}}(s)\right|_{s=0},
$$

where $\operatorname{Tr}$ denotes summation over $v^{a}$. The dimensionless quantities

$$
\lambda=\lambda / \mu, \quad B=B / \mu^{2}, \quad R=R \mu
$$

have been introduced using the auxiliary renormalization scale $\mu$. The zeta function in Eq. (19) can be found via analytical continuation to $s \rightarrow 0$ in the complex plane, and the final expression for $\delta U^{\text {gh }}$ is (see Ref. [33])

$$
\begin{aligned}
\delta U^{\mathrm{gh}}(B, R)= & -4 \sum_{k=1}^{\infty} k\left[\sum_{m=-\frac{k-1}{2}}^{\frac{k-1}{2}} \Psi_{\mathrm{gh}}\left(k, m, 0, \frac{\sqrt{3} B}{2}, R\right)-\frac{3}{4} B^{2} R^{4} \frac{1}{48}\left(1-\frac{1}{k}+\frac{1}{k^{2}}\right)\right] \\
& +\frac{B^{2} R^{4}}{48}(2-3 \gamma+3 \log 2-3 \log R)-\frac{3 B^{4} R^{8}}{10240}, \\
\Psi^{\mathrm{gh}}(k, m, t, \breve{B}, R)= & \log \frac{\exp \left(-\frac{\breve{b} R^{2}}{4}\right) M\left(\frac{k+1}{2}-m+\frac{t^{2}}{2 \breve{B}}, k+1, \frac{\breve{B} R^{2}}{2}\right)}{k !\left(\frac{t R}{2}\right)^{-k} I_{k}(t R)} .
\end{aligned}
$$

The sums in Eq. (21) converge and can be computed numerically.

The ghost contribution $F^{\mathrm{gh}}(B, R)=\delta U^{\mathrm{gh}}(B, R) / V_{R}$ to the free energy density defined by Eq. (21) is shown in Fig. 3. It demonstrates expected behavior in both the field strength $B$ and domain size $R$. In the infinitevolume limit it approaches the expected one-loop ghost contribution.

\section{B. Gauge-field fluctuations}

Eigenvalues of the relevant gluon operator

$$
-\breve{D}^{2} \delta_{\mu \nu}+2 i \breve{B}_{\mu \nu}
$$

differ from the ghost eigenvalues by an overall shift due to the term $2 i \breve{B}_{\mu \nu}$. Though in the infinite space-time this shift leads to the presence of infinitely many exact gluon zero modes, for $R<\infty$ the degeneracy of these modes disappears, and all corresponding eigenvalues become positive.

This subset of modes will be referred to below as quasizero modes. Since exact zero modes are absent for $R<\infty$, one may naively expect that plain one-loop calculation should suffice for moderate values of the domain size $R$. The calculation proceeds completely analogously to the computation in the previous subsection, and one finds the following result for $\delta U^{\mathrm{gl}}$ (see Ref. [33] for details): 

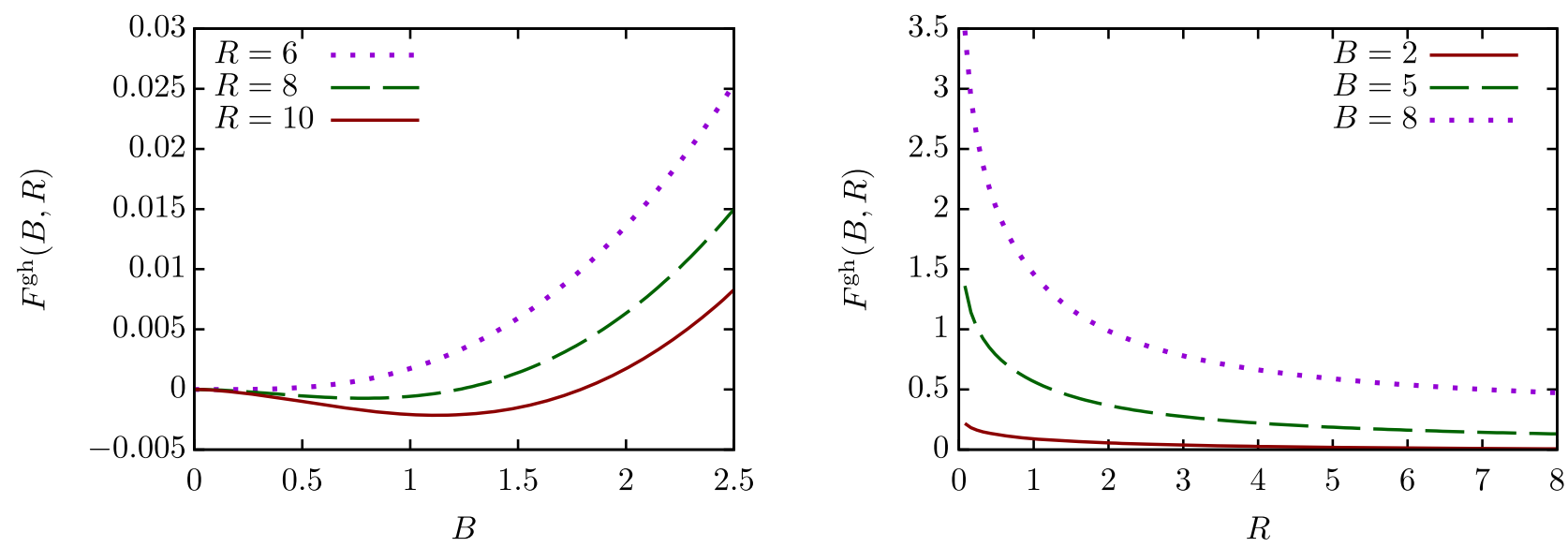

FIG. 3. Contribution of the Faddeev-Popov determinant to the free energy density. The domain size $R$ and field strength $B$ are given in units of the renormalization scale $\mu$ [Eq. (20)]. If $R$ is sufficiently large, then a minimum exists at nonzero field strength $B$. For any $B$ the ghost contribution is minimal in the infinite-volume limit $R \rightarrow \infty$.

$$
\begin{aligned}
\delta U^{\mathrm{gl}}(B, R)= & 4 \sum_{k=1}^{\infty} k\left[\sum_{m=-\frac{k-1}{2}}^{\frac{k-1}{2}} \Psi_{\mathrm{gl}}\left(k, m, 0, \frac{\sqrt{3} B}{2}, R\right)-\frac{3}{4} B^{2} R^{4} \frac{1}{24}\left(1-\frac{1}{k}-\frac{5}{k^{2}}\right)\right] \\
& -\frac{B^{2} R^{4}}{48}(31+30 \gamma-30 \log 2+30 \log R)+\frac{3 B^{4} R^{8}}{5120}, \\
\Psi^{\mathrm{gl} l}(k, m, t, B, R)= & \log \frac{\exp \left(-\frac{B R^{2}}{2}\right) M\left(\frac{k+1}{2}-m+1+\frac{t^{2}}{2 B}, k+1, \frac{B R^{2}}{2}\right) M\left(\frac{k+1}{2}-m-1+\frac{t^{2}}{2 B}, k+1, \frac{B R^{2}}{2}\right)}{\left(k !\left(\frac{t R}{2}\right)^{-k} I_{k}(t R)\right)^{2}} .
\end{aligned}
$$

The free energy density, as given by Eq. (23), is shown in Fig. 4. One would expect that the character of the strongfield limit should be insensitive to the presence of the boundary, since it corresponds to short distances. Meanwhile, the free energy density decreases without bound at large $B$ and fixed $R$, which does not comply with known results $[5,8]$ and, more generally, with asymptotic freedom. It also does not approach a constant at large
$R$ and fixed $B$, which implies the absence of a sensible thermodynamic limit in the system. This behavior is due to the manifestation of infinitely many quasizero eigenvalues that tend to zero as the dimensionless quantity $B R^{2}$ increases (see Fig. 5), which occurs both in the strongfield and thermodynamic limits.

If all eigenvalues are sufficiently large to provide Gaussian damping in the functional integral (at small
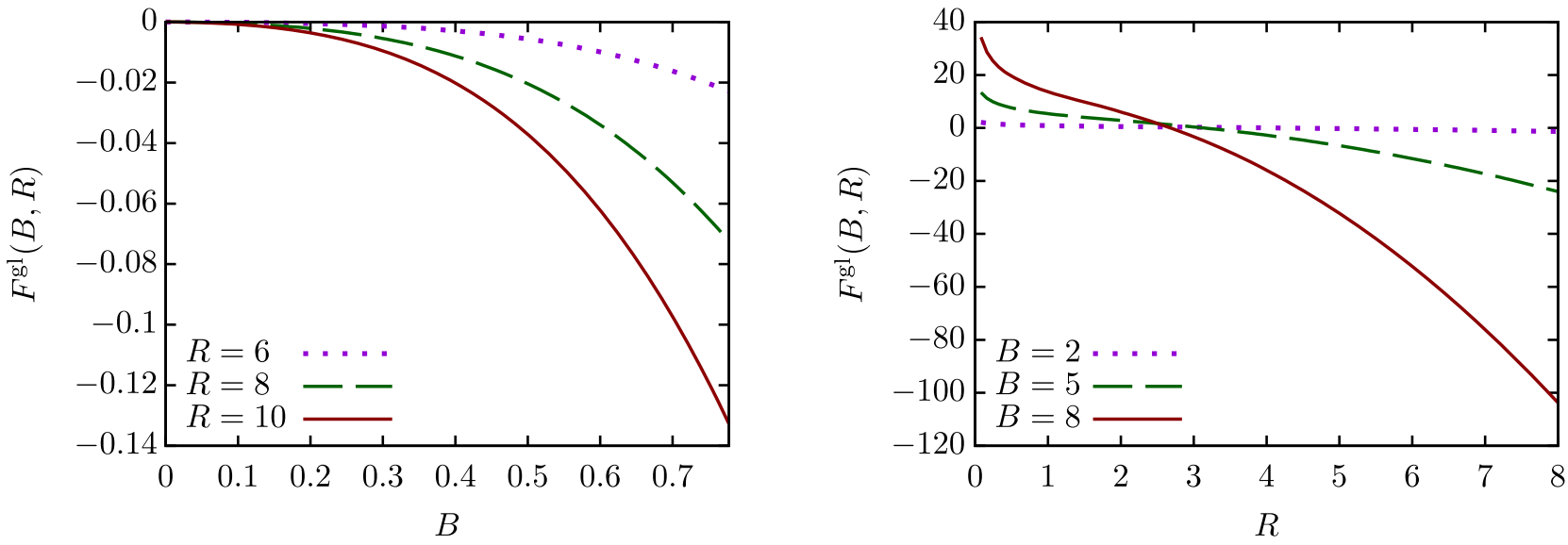

FIG. 4. Dependence of the one-loop gluon contribution to the free energy density on $B$ and $R$ [in units of the renormalization scale $\mu$; see Eq. (20)]. The strong-field and large-volume limits are incorrect due to the presence of infinitely many gluon quasizero modes. 


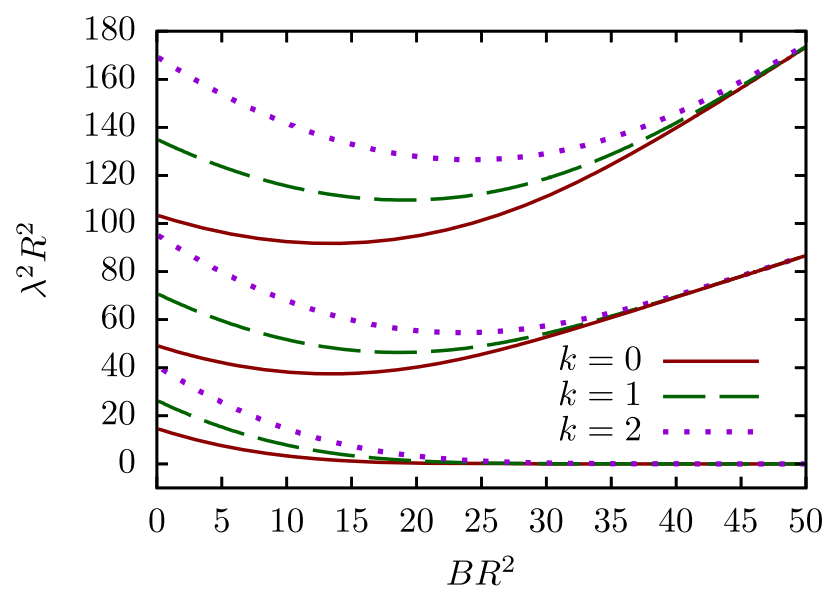

FIG. 5. Dependence of gluon eigenvalues [see Eq. (A1)] on $B R^{2}$. In the limit $B R^{2} \rightarrow \infty$, an infinite number of zero modes emerge.

$B R^{2}$ ), then Eq. (17) can be considered as justified. The smaller the eigenvalues, the worse the one-loop approximation becomes, and finally it results in a strong-field limit that is upside down. It becomes clear that one has to take into account the mixing between normal and quasizero modes, which means going beyond the one-loop approximation of the free energy at large $B R^{2}$. The calculation of the effective potential for an Abelian self-dual field in infinite space-time gives a guiding prescription [8], based on the observation that if one evaluates the functional integral over normal (nonzero) modes first and accounts for their interaction with zero modes, then the obtained effective action has a finite quadratic-in-zero-modes part. In other words, due to the interactions, zero modes gain an effective "mass" $\mu_{0}^{2}=\bar{\varkappa} B$, which provides an appropriate Gaussian measure. Here $\bar{x}$ is a constant. Schematically, the contribution to effective "mass" is shown in Fig. 6. The filled circles denote all possible diagrams which include the propagators of normal gluon and quark modes. For $S U(2)$ gluodynamics the lowest-order value of the zero mode "effective mass" takes the value [8]

$$
\mu_{0}^{2}=\bar{\varkappa} B, \quad \bar{\varkappa}=\frac{g^{2}}{24 \pi^{2}} .
$$

The final result for the free energy density in the infinitevolume limit agrees completely with the renormalization group estimate [5] and, as it should be, with asymptotic freedom [8].
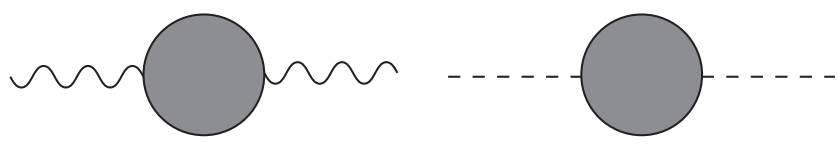

FIG. 6. The wavy line corresponds to gluon quasizero modes, while the dashed line corresponds to quark quasizero modes. The filled circles denote all possible diagrams with a normal quark and gluon modes.
For the finite-volume case, this observation suggests that, in particular, the interaction of normal modes with quasizero modes generates a shift

$$
\begin{aligned}
\bar{\lambda}_{\mathrm{eff}}^{2}(B, R) & =\bar{\lambda}^{2}(B, R)+\varkappa\left(B R^{2}\right) B, \\
\lim _{R \rightarrow \infty} \bar{\lambda}^{2}(B, R) & =0, \quad \lim _{z \rightarrow \infty} \varkappa(z)=\bar{\varkappa}>0,
\end{aligned}
$$

where $\bar{\lambda}^{2}(B, R)$ are quasizero eigenvalues of the operator (22), and the function $\varkappa\left(B R^{2}\right)$ should approach $\bar{\varkappa}$ in both the infinite-volume and strong-field limits.

The normal mode propagators that are incorporated into the diagrams in Fig. 6 can be represented at most as an infinite series over quantum numbers of the modes. Truncation of these series is unreliable since, in general, diagrams are UV divergent. Given that, the calculation of the dependence of $x$ on the dimensionless quantity $z=B R^{2}$ appears to be an extremely complicated task, even at the lowest perturbation order. Moreover, in the absence of a small expansion parameter, the perturbative expansion cannot lead to a decisive result anyway.

Meanwhile, it seems to be possible to identify the general form of $\varkappa(z)$ suitable for a qualitative estimate of the available fundamentally different dependencies of the free energy density on the domain size. Two restrictions for the function $\varkappa(z)$ can be identified. The first one is given by Eq. (24). As has already been noted, it follows from the existence of the thermodynamic limit and agreement with asymptotic freedom and the strong-field limit. Another restriction,

$$
\lim _{z \rightarrow 0} \varkappa(z)=0
$$

follows from the scaling of all eigenvalues at small $R$,

$$
\lim _{R \rightarrow 0} \lambda^{2}(z) \propto \lim _{R \rightarrow 0} 1 / R^{2}=0
$$

which means that corrections to the effective action of quasizero modes coming from the diagrams (Fig. 6) are expected to vanish, in analogy with decoupling of the infinitely heavy particles.

A trial function $\varkappa(z)$ can be taken in the form

$$
\begin{aligned}
\varkappa(z)= & \frac{2}{\pi}\left[\arctan \exp \left(\frac{z-z_{0}}{a}\right)+\arctan \exp \left(\frac{z_{0}-z}{a}\right)\right. \\
& \left.-2 \arctan \exp \left(-\frac{z_{0}}{a}\right)\right],
\end{aligned}
$$

which, in addition to the above restrictions at $z \rightarrow 0$ and $z \rightarrow \infty$, also reflects a change in the behavior of eigenvalues at a certain value of $z$, when all normal eigenvalues start to increase as $R$ decreases; see Fig. 5. The function $\varkappa$ is plotted in Fig. 7. 
Incorporation of the effective "mass" $\varkappa\left(B R^{2}\right)$ leads to the following gluon contribution to the effective action (the procedure described in Ref. [33] is refined in Appendix A):

$$
\begin{aligned}
\delta U_{\varkappa}^{\mathrm{gl}}(B, R)= & 4 \sum_{k=1}^{\infty} k\left[\sum_{m=-\frac{k-1}{2}}^{\frac{k-1}{2}} \Psi \mathrm{gl}\left(k, m, 0, \frac{\sqrt{3} B}{2}, R\right)-\frac{3}{4} B^{2} R^{4} \frac{1}{24}\left(1-\frac{1}{k}-\frac{5}{k^{2}}\right)\right] \\
& -\frac{B^{2} R^{4}}{48}(31+30 \gamma-30 \log 2+30 \log R)+\frac{3 B^{4} R^{8}}{5120} \\
& +4 \sum_{k=0}^{\infty}\left[(k+1) \log \frac{\lambda_{\uparrow k_{2}^{k} 0}^{2}(B, R)+\varkappa\left(B R^{2}\right) B}{\lambda_{\uparrow k_{2}^{k} 0}^{2}(B, R)}+(k+1) \log \frac{\lambda_{\downarrow k \frac{-k}{2} 0}^{2}(B, R)-\varkappa\left(B R^{2}\right) B}{\lambda_{\downarrow k \frac{-k}{2} 0}^{2}(B, R)}\right] .
\end{aligned}
$$

The corresponding free energy density is plotted in Fig. 8. The correct behavior of the free energy density for $B R^{2} \rightarrow \infty$, consistent with asymptotic freedom and the existence of the thermodynamic limit, is restored. It is seen that the free energy density acquires a minimum at intermediate values of field strength and domain size.

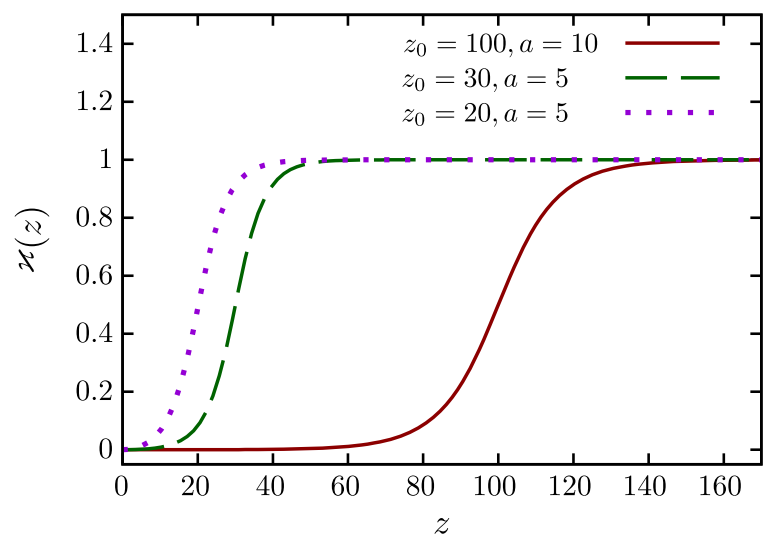

FIG. 7. Profile of the function $\varkappa(z)$ used in Fig. 8.

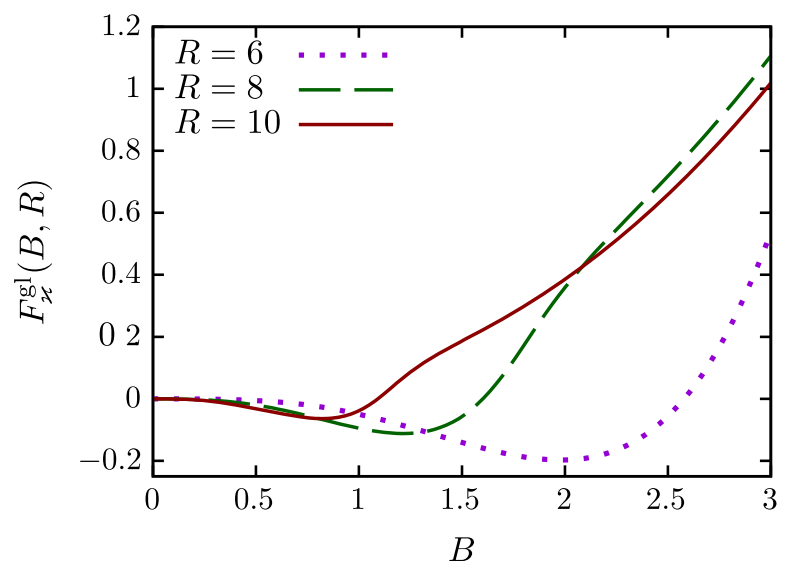

The free energy density for pure $S U(3)$ gluodynamics for a finite domain of Abelian (anti-)self-dual gluon fields is given by the sum of Eqs. (21) and (27). The result is iillustrated on the last figure in Sec. III.C. The existence of the minimum of the free energy density as a function of two variables is clearly seen.

\section{Quark contribution}

To complete the calculation for full QCD with massless quarks, we have to study the quark contribution to the effective potential

$$
\begin{aligned}
\delta U^{\mathrm{q}}(B, R) & =-N_{f} \operatorname{Tr} \ln \frac{i \not D}{\left.i \not D\right|_{B=0}} \\
& =-N_{f} \sum_{k, j, n} \operatorname{Tr} \ln \frac{i \lambda_{k j n}(B, R)}{i \lambda_{k j n}(0, R)}=\left.N_{f} \frac{d}{d s} \zeta^{\mathrm{q}}(s)\right|_{s=0},
\end{aligned}
$$

where $N_{f}$ is the number of quark flavors, and $\lambda(B, R)$ are eigenvalues of the Dirac operator in a spherical domain of radius $R$ with a homogeneous Abelian (anti-)self-dual field and bag boundary condition (see Appendix B).

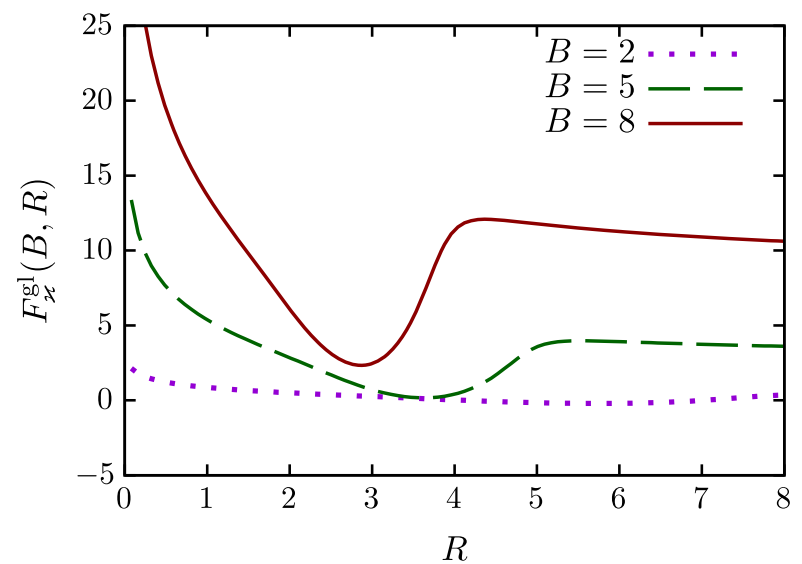

FIG. 8. Improved gluon contribution to the free energy with the effective "mass" $\varkappa$ given by Eq. (26). The parameters of $\varkappa$ are taken such that the minimum is well pronounced: $z_{0}=100$ and $a=10$. All quantities are measured in units of the renormalization scale $\mu$ [Eq. (20)]. 
The zeta function $\zeta^{\mathrm{q}}(s)$ can be split into two parts [48],

$$
\begin{aligned}
\zeta^{\mathrm{q}}(s) & =\cos (\pi s) \zeta_{\not D^{2}}\left(\frac{s}{2}\right)-i \sin (\pi s) \eta(s), \\
\left.\frac{d}{d s} \zeta^{\mathrm{q}}(s)\right|_{s=0} & =\frac{1}{2} \zeta_{\not \not^{2}}^{\prime}(0)-i \pi \eta(0) .
\end{aligned}
$$

For the purpose of the present study we only need $\zeta_{\not p^{2}}$. The parity-odd term $\eta(s)$ contributes to the imaginary part of the effective potential and can be related to the $U_{\mathrm{A}}(1)$ anomalous breakdown [26]. The parity-even part $\zeta_{\not p^{2}}$ can be written in the following form:

$$
\begin{aligned}
\zeta_{\not b^{2}}(s) & =\operatorname{Tr} \frac{\sin \pi s}{\pi} \sum_{k=0}^{\infty} \sum_{j_{3}=-\frac{k+1}{2}}^{\frac{k+1}{2}}(k+1) \int_{0}^{\infty} \frac{d t}{t^{2 s}} \frac{d}{d t} \Psi \mathrm{q}\left(k+1, j_{3}, t, \hat{B}, R\right), \\
\Psi^{\mathrm{q}}(k+1, j, t, \hat{B}, R) & =\log \frac{A\left(-i t, k, j_{3}, \hat{B}, R\right) A\left(i t, k, j_{3}, \hat{B}, R\right)}{A\left(-i t, k, j_{3}, 0, R\right) A\left(i t, k, j_{3}, 0, R\right)},
\end{aligned}
$$

where $A(\lambda, k, j, B, R)=0$ is the equation for eigenvalues. Proceeding in the same manner as in the previous section (see Appendix $\mathrm{C}$ for details), we arrive at the expression

$$
\begin{aligned}
\delta U^{\mathrm{q}}(B, R)= & N_{f}\left\{-\frac{1}{2} \operatorname{Tr} \sum_{k=1}^{\infty}(k+1)\left[\sum_{j=-\frac{k-1}{2}}^{\frac{k-1}{2}} \Psi \mathrm{q}(k, j, 0, \hat{B}, R)-\hat{B}^{2} R^{4} \frac{1}{12}\left(1-\frac{2}{k^{2}}\right)\right]\right. \\
& \left.+\frac{B^{2} R^{4}}{144}\left(5+6 \gamma-6 \log 2+\pi^{2}+6 \log R\right)-B^{4} R^{8} \frac{1}{30720}\right\} .
\end{aligned}
$$

Due to the quark quasizero modes (see Fig. 9), the quark contribution to the free energy density given by Eq. (30) and shown in Fig. 10 exhibits the inconsistency with the correct strong-field and thermodynamic limits, similar to inconsistencies in the gluon effective potential given by Eq. (23) and illustrated in Fig. 4.

Following reasoning analogous to the case of gluons in the previous subsection, the improved calculation of the quark contribution has to take into account the generation of the effective "mass" for quasizero eigenmodes of quarks due to the interaction of quark quasizero modes with normal gluon and normal quark modes (see the right-hand side diagram in Fig. 6). The improved quark contribution reads

$$
\begin{aligned}
\delta U_{\varkappa}^{\mathrm{q}}(B, R)= & N_{f}\left\{-\frac{1}{2} \operatorname{Tr} \sum_{k=1}^{\infty}(k+1)\left[\sum_{j_{3}=-\frac{k-1}{2}}^{\frac{k-1}{2}} \Psi \mathrm{q}(k, j, 0, \hat{B}, R)-\hat{B}^{2} R^{4} \frac{1}{12}\left(1-\frac{2}{k^{2}}\right)\right]\right. \\
& \left.+\frac{B^{2} R^{4}}{144}\left(5+6 \gamma-6 \log 2+\pi^{2}+6 \log R\right)-B^{4} R^{8} \frac{1}{30720}\right\} \\
& -\frac{N_{f}}{2} \operatorname{Tr} \sum_{k=0}^{\infty}\left[(k+1) \log \frac{\lambda_{k \frac{k}{2} 0}^{2}(B, R)+\varkappa\left(B R^{2}\right) B}{\lambda_{k_{2} 0}^{2}(B, R)}+(k+1) \log \frac{\lambda_{k \frac{-k}{2} 0}^{2}(B, R)-\varkappa\left(B R^{2}\right) B}{\lambda_{k \frac{k}{2} 0}^{2}(B, R)}\right],
\end{aligned}
$$

similarly to Eq. (27). The effective potential is shown in Fig. 11. 
Combining the ghost, improved gluon, and quark contributions, one finds

$$
\begin{aligned}
& \delta U_{\varkappa}(B, R)=\delta U_{\varkappa}^{\mathrm{gl}}(B, R)+\delta U^{\mathrm{gh}}(B, R)+\delta U_{\varkappa}^{\mathrm{q}}(B, R) \\
& =4 \sum_{k=1}^{\infty} k\left[\sum_{m=-\frac{k-1}{2}}^{\frac{k-1}{2}} \Psi \mathrm{gl}\left(k, m, 0, \frac{\sqrt{3} B}{2}, R\right)-\frac{3}{4} B^{2} R^{4} \frac{1}{24}\left(1-\frac{1}{k}-\frac{5}{k^{2}}\right)\right] \\
& +4 \sum_{k=0}^{\infty}\left[(k+1) \log \frac{\lambda_{\mathrm{gl}, k \frac{k}{2} 0}^{2}(B, R)+\varkappa\left(B R^{2}\right) B}{\lambda_{\mathrm{gl}, k \frac{k}{2} 0}^{2}(B, R)}+(k+1) \log \frac{\lambda_{\mathrm{gl}, k \frac{k k_{0}}{2}}^{2}(B, R)-\varkappa\left(B R^{2}\right) B}{\lambda_{\mathrm{gl}, k \frac{k}{2} 0}^{2}(B, R)}\right] \\
& -4 \sum_{k=1}^{\infty} k\left[\sum_{m=-\frac{k-1}{2}}^{\frac{k-1}{2}} \Psi^{\mathrm{gh}}\left(k, m, 0, \frac{\sqrt{3} B}{2}, R\right)-\frac{3}{4} B^{2} R^{4} \frac{1}{48}\left(1-\frac{1}{k}+\frac{1}{k^{2}}\right)\right] \\
& -\frac{N_{f}}{2} \operatorname{Tr} \sum_{k=1}^{\infty}(k+1)\left[\sum_{j_{3}=-\frac{k-1}{2}}^{\frac{k-1}{2}} \Psi \mathrm{q}(k, j, 0, \hat{B}, R)-\hat{B}^{2} R^{4} \frac{1}{12}\left(1-\frac{2}{k^{2}}\right)\right] \\
& -\frac{N_{f}}{2} \operatorname{Tr} \sum_{k=0}^{\infty}\left[(k+1) \log \frac{\lambda_{\mathrm{q}, k_{2}^{k} 0}^{2}(B, R)+\varkappa\left(B R^{2}\right) B}{\lambda_{\mathrm{q}, k_{2}^{k} 0}^{2}(B, R)}+(k+1) \log \frac{\lambda_{\mathrm{q}, k \frac{k-k}{2} 0}^{2}(B, R)-\varkappa\left(B R^{2}\right) B}{\lambda_{\mathrm{q}, k \frac{k}{2} 0}^{2}(B, R)}\right] \\
& -\frac{B^{2} R^{4}}{48}(29+33 \gamma-33 \log 2+33 \log R)+\frac{3 B^{4} R^{8}}{10120}+N_{f}\left\{\frac{B^{2} R^{4}}{144}\left(5+6 \gamma-6 \log 2+\pi^{2}+6 \log R\right)-\frac{B^{4} R^{8}}{30720}\right\} \text {. }
\end{aligned}
$$

The corresponding free energy density is shown on the right-hand side of Fig. 12. The total free energy density demonstrates a well-pronounced minimum as a function of field strength and domain size. The quark contribution does not change the result of pure gluodynamics qualitatively, though the field strength at the minimum is considerably reduced.

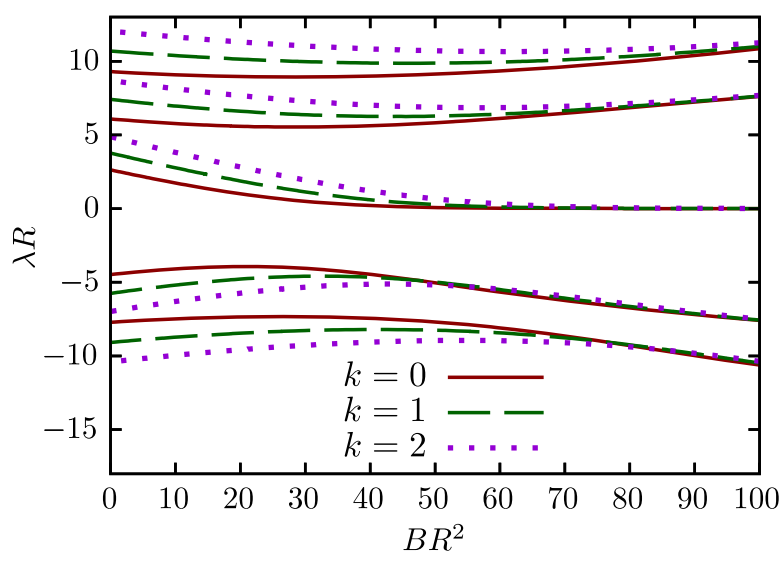

FIG. 9. Dependence of quark eigenvalues on $B R^{2}$. At $B R^{2} \rightarrow \infty$, an infinite number of zero modes emerge. Normal modes come in positive-negative pairs $\lambda^{ \pm}$. Note that $\lambda^{+} \not \neq-\lambda^{-}$for finite $B R^{2}$, but the equality is restored in the limit $B R^{2} \rightarrow \infty$. An infinite number of quasizero modes are not chiral for finite $R$ and become chiral zero modes for asymptotically large $B R^{2}$.

\section{One-loop beta function}

The effective action $U=U^{\mathrm{cl}}+\delta U_{\varkappa}$ should not depend on the renormalization scale $\mu[49,50]$, that is,

$$
\mu \frac{d}{d \mu} U=0
$$

with the classical action $\left(V_{R}\right.$ is a four-dimensional ball of radius $R$ )

$$
U^{\mathrm{cl}}=\int_{V_{R}} d^{4} x \frac{1}{4 g^{2}}\left(B_{\mu \nu}^{a}\right)^{2}=\frac{\pi^{2} B^{2} R^{4}}{2 g^{2}}
$$

and $\delta U_{\varkappa}$ is given by Eq. (32). Since only terms containing $\log R$ contribute to Eq. (33), one obtains

$$
\mu \frac{d}{d \mu}\left[\frac{\pi^{2} B^{2} R^{4}}{2 g^{2}}+\frac{B^{2} R^{4}}{16}\left(-11+\frac{2}{3} N_{f}\right) \log R\right]=0,
$$

which is equivalent to the equation

$$
\mu \frac{d}{d \mu} g=-\frac{g^{3}}{16 \pi^{2}}\left(11-\frac{2}{3} N_{f}\right)
$$

exposing the correct one-loop $\beta$ function of QCD with $N_{f}$ quark flavors. 

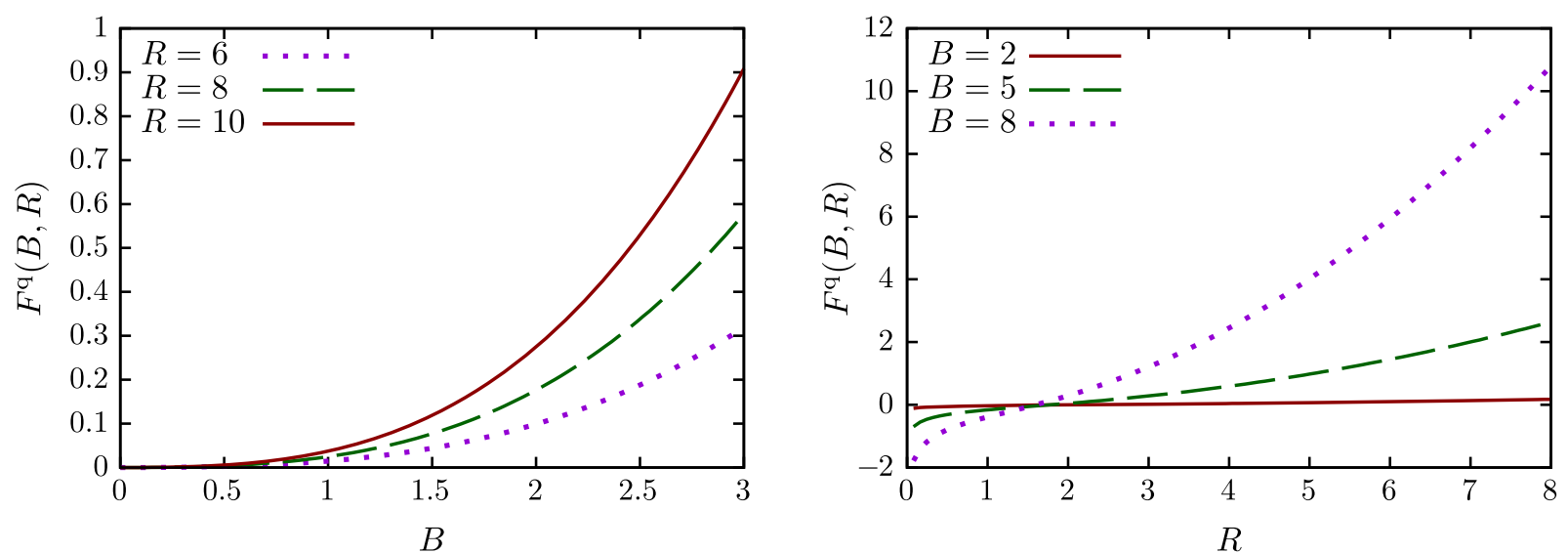

FIG. 10. Dependence of the one-loop quark contribution to the free energy density on $R$ and $B, N_{f}=1$. All quantities are given in units of the renormalization scale $\mu$ [Eq. (20)]. The strong-field and large-size regimes are incorrect.
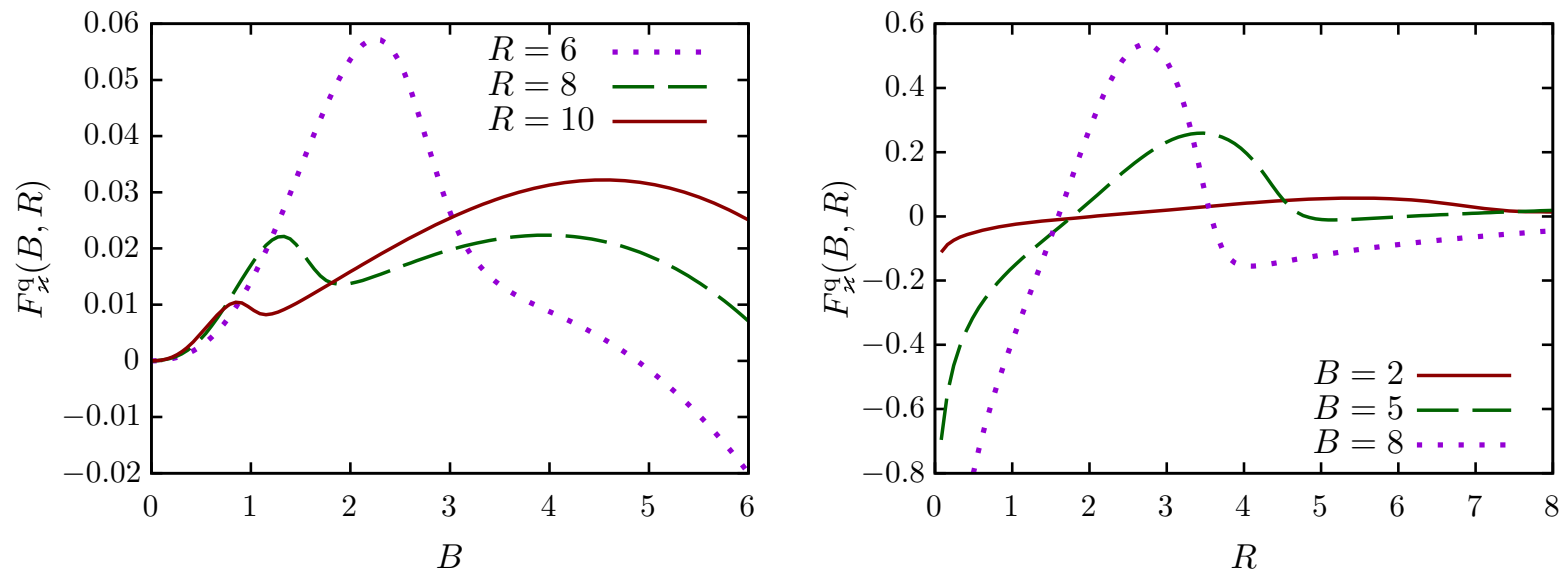

FIG. 11. Improved quark contribution to the free energy density $R$ and $B, N_{f}=1$, in units of the renormalization scale $\mu$ [Eq. (20)]. The function $\varkappa$ is given by Eq. (26) $\left(z_{0}=100, a=10\right)$. The correct strong-field and large-size limits are restored.
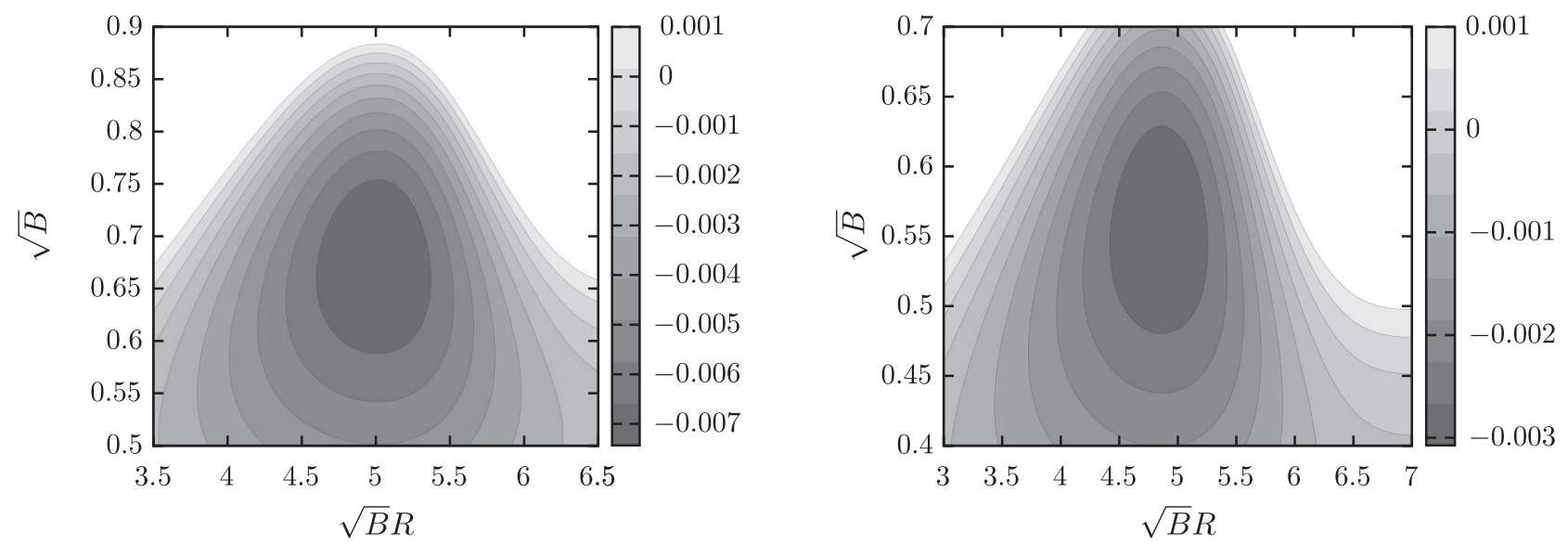

FIG. 12. Total quantum correction to the free energy density $F_{\varkappa}(B, R)$ given by Eq. (32) with $\varkappa$ given by Eq. (26) $\left(z_{0}=30, a=5\right)$. The left plot is for pure gluodynamics $\left(N_{f}=0\right)$, while the right plot is for full QCD with two massless quark flavors $\left(N_{f}=2\right)$, in units of the renormalization scale $\mu$. 


\section{DISCUSSION}

We have studied, as far as it has been possible with analytical methods, the influence of finite-size effects on the vacuum free energy density of full QCD with $N_{f}$ massless flavors in the presence of a homogeneous (anti-) self-dual Abelian background gluon field. The most essential result is illustrated on the right-hand side Fig. 12, where the quantum correction to the zero-temperature free energy density of the four-dimensional spherical domain is plotted as a function of the background field strength $B$ and domain radius $R$. It indicates that the quantum correction to the free energy density may have a minimum at finite values of $B$ and $R$. In the domain wall network representation of the vacuum mean field, the existence of this minimum means that in the statistically dominant networks the individual domains should have a finite size varying near the mean value, and infinite growth of individual domains is prohibited by the minimization of the overall free energy of the configuration. This suggests that the domain wall ensemble should include an infinite number of configurations with degenerate energy.

The character of this energy-driven disorder strongly depends on the details of the behavior of the free energy density in the vicinity of the minimum. One may expect that a shallow and flat profile near the minimum may lead to strong variations of the geometrical shape as well as deviations of the field strength from the mean value, which will allow the presence of highly irregular networks among the dominant configurations, characterized by the strong entanglement of domains, etc. A deep and steep profile would assume that spatially periodic networks should dominate, bringing the long-range (periodic) order into the mean-field configurations.

The result of the present paper, Fig. 12, certainly has the status of a preliminary rough estimate, since the validity of the one-loop approximation is indeterminate, especially due to the indefiniteness of the treatment of the interaction between quasizero and normal modes. Though domain surface effects are not expected to overcome the bulk properties of the free energy density, a complete analysis requires also accounting for the surface free energy. A straightforward numerical calculation, within the lattice approximation, for instance, could be useful. A difficulty for lattice calculations can be caused by the nonstandard boundary conditions, which have to represent the physical boundary of a domain. However, this possibility does not look hopeless in view of the recent lattice
QCD calculations for rotating strongly interacting matter, where Dirichlet and Neumann boundary conditions have been implemented [51,52].

\section{ACKNOWLEDGMENTS}

We acknowledge useful discussions with Michael Bordag, Irina Pirozhenko, Victor Braguta, and Artem Roenko.

\section{APPENDIX A: GLUON QUASIZERO MODES}

\section{Contribution of gluon quasizero modes to the effective potential}

In this section, we calculate the contribution of gluon quasizero modes $\lambda_{\uparrow k \frac{k}{2} 0}$ to the effective potential. These modes correspond to the smallest-magnitude solutions to the equations

$M\left(-\frac{\lambda^{2}}{2|\breve{B}|}, k+2, \frac{|\breve{B}| R^{2}}{2}\right)=0, \quad k=0,1,2, \ldots$,

which reduce to

$$
\left(\frac{\lambda R}{2}\right)^{-k-1} J_{k+1}(\lambda R)=0, \quad k=0,1, \ldots
$$

at $B \rightarrow 0$. The contribution of gluon quasizero modes to the effective potential can be expressed as

$$
\begin{aligned}
\delta U^{\mathrm{gl}(0)}(B, R) & =\frac{1}{2} \operatorname{Tr} \log \frac{\lambda_{\uparrow k_{2}^{k} 0}^{2}(B, R)}{\lambda_{k 0}^{2}(0, R)}=-\left.\frac{1}{2} \frac{d}{d s} \zeta^{\mathrm{gl}(0)}(s)\right|_{s=0}, \\
\zeta^{\mathrm{gl}(0)}(s) & =2 \operatorname{Tr} \sum_{k=0}^{\infty}(k+1)\left(\lambda_{\uparrow k_{2}^{-2} 0}^{-2 s}(B, R)-\lambda_{k 0}^{-2 s}(0, R)\right),
\end{aligned}
$$

where the factor of 2 in the definition of $\zeta^{\mathrm{gl}(0)}(s)$ originates from the two polarizations of quasizero gluon modes, and the color trace yields a factor of 4 . For the sake of brevity, we omit the color eigenvalue $v_{a}=\sqrt{3} / 2$ and restore it in the final answer $\left(B \rightarrow v_{a} B\right)$.

To continue $\zeta^{\mathrm{gl}(0)}(s)$ to $s \rightarrow 0$, we add and subtract several terms of the asymptotic expansion in $k$ found with the help of Eq. (D5),

$$
\begin{aligned}
\zeta^{\mathrm{gl}(0)}(s)= & 8 \sum_{k=0}^{\infty}\left[(k+1)\left(\lambda_{\uparrow k_{2}^{k} 0}^{-2 s}(B, R)-\lambda_{k 0}^{-2 s}(0, R)\right)\right. \\
& \left.-(k+1)^{-2 s} R^{2 s}\left(B R^{2} s-2 \alpha B R^{2} s(1+s)(k+1)^{-2 / 3}+B R^{2} \frac{s}{4}\left(4+B R^{2}(1+2 s)\right)(k+1)^{-1}\right)\right] \\
& +8 \sum_{k=0}^{\infty}(k+1)^{-2 s} R^{2 s}\left(B R^{2} s-2 \alpha B R^{2} s(1+s)(k+1)^{-2 / 3}+B R^{2} \frac{s}{4}\left(4+B R^{2}(1+2 s)\right)(k+1)^{-1}\right) .
\end{aligned}
$$


The first sum is an analytic function for $\Re s>0$. The second sum is evaluated for $\mathfrak{R} s>1 / 2$ and analytically continued to $s \rightarrow 0$ :

$$
\begin{aligned}
& \sum_{k=0}^{\infty}(k+1)^{-2 s} R^{2 s}\left(B R^{2} s-2 \alpha B R^{2} s(1+s)(k+1)^{-2 / 3}+B R^{2} \frac{s}{4}\left(4+B R^{2}(1+2 s)\right)(k+1)^{-1}\right) \\
& =B R^{2+2 s} s(\zeta(2 s)-2 \alpha(1+s) \zeta(2 / 3+2 s)+\zeta(1+2 s))+B^{2} R^{4+2 s} \frac{s}{4}(1+2 s) \zeta(1+2 s) .
\end{aligned}
$$

Finally, we obtain

$$
\begin{aligned}
\delta U^{\mathrm{gl}(0)}(B, R)= & -\left.\frac{1}{2} \frac{d}{d s} \zeta^{\mathrm{gl}(0)}(s)\right|_{s=0} \\
= & -4 \sum_{k=0}^{\infty}\left[-(k+1) \log \frac{\lambda_{\uparrow k_{2}^{k} 0}^{2}(B, R)}{\lambda_{k 0}^{2}(0, R)}-B R^{2}\left(1-2 \alpha(k+1)^{-2 / 3}+(k+1)^{-1}\right)-\frac{B^{2} R^{4}}{4(k+1)}\right] \\
& -2 B R^{2}\left[-1+2 \gamma+2 \log R-4 \alpha \zeta\left(\frac{2}{3}\right)\right]-B^{2} R^{4}(1+\gamma+\log R) .
\end{aligned}
$$

\section{Contribution of quasizero modes with the effective "mass"}

If one includes the effective "mass" $\varkappa$ for quasizero modes in the considerations of the previous section, the formulas become

$$
\begin{aligned}
\delta U_{\varkappa}^{\mathrm{gl}(0)}(B, R) & =\frac{1}{2} \operatorname{Tr} \log \frac{\lambda_{\uparrow k_{2}^{k} 0}^{2}(B, R)+\varkappa\left(B R^{2}\right) B}{\lambda_{k 0}^{2}(0, R)}=-\left.\frac{1}{2} \frac{d}{d s} \zeta^{\mathrm{gl}(0)}(s)\right|_{s=0}, \\
\zeta_{\varkappa}^{\mathrm{gl}(0)}(s) & =2 \operatorname{Tr} \sum_{k=0}^{\infty}(k+1)\left(\left(\lambda_{\uparrow k_{2}^{2} 0}^{2}(B, R)+\varkappa\left(B R^{2}\right) B\right)^{-s}-\lambda_{k 0}^{-2 s}(0, R)\right) .
\end{aligned}
$$

In analogy to the previous section,

$$
\begin{aligned}
\zeta_{\varkappa}^{\mathrm{gl}(0)}(s)= & 8 \sum_{k=0}^{\infty}\left[(k+1)\left(\left(\lambda_{\uparrow k_{2}^{k} 0}^{2}(B, R)+\varkappa\left(B R^{2}\right) B\right)^{-s}-\lambda_{k 0}^{-2 s}(0, R)\right)\right. \\
& \left.-(k+1)^{-2 s} R^{2 s}\left(B R^{2} s-2 \alpha B R^{2} s(1+s)(k+1)^{-2 / 3}+B R^{2} \frac{s}{4}\left(4+B R^{2}(1+2 s)-4 \varkappa\left(B R^{2}\right)\right)(k+1)^{-1}\right)\right] \\
& +8 \sum_{k=0}^{\infty}(k+1)^{-2 s} R^{2 s}\left(B R^{2} s-2 \alpha B R^{2} s(1+s)(k+1)^{-2 / 3}+B R^{2} \frac{s}{4}\left(4+B R^{2}(1+2 s)-4 \varkappa\left(B R^{2}\right)\right)(k+1)^{-1}\right)
\end{aligned}
$$

The first sum is an analytic function for $\mathfrak{R} s>0$. The second sum is evaluated for $\mathfrak{R} s>1 / 2$ and analytically continued to $s \rightarrow 0$ :

$$
\begin{aligned}
& \sum_{k=0}^{\infty}(k+1)^{-2 s} R^{2 s}\left(B R^{2} s-2 \alpha B R^{2} s(1+s)(k+1)^{-2 / 3}+B R^{2} \frac{s}{4}\left(4+B R^{2}(1+2 s)-4 \varkappa\left(B R^{2}\right)\right)(k+1)^{-1}\right) \\
& \quad=B R^{2+2 s} s\left(\zeta(2 s)-2 \alpha(1+s) \zeta(2 / 3+2 s)+\left(1-\varkappa\left(B R^{2}\right)\right) \zeta(1+2 s)\right)+B^{2} R^{4+2 s} \frac{s}{4}(1+2 s) \zeta(1+2 s) .
\end{aligned}
$$

Finally, 


$$
\begin{aligned}
\delta U_{\varkappa}^{\mathrm{gl}(0)}(B, R)= & -\left.\frac{1}{2} \frac{d}{d s} \zeta_{\varkappa}^{\mathrm{gl}(0)}(s)\right|_{s=0} \\
= & -4 \sum_{k=0}^{\infty}\left[-(k+1) \log \frac{\lambda_{\uparrow k \frac{k}{2} 0}^{2}(B, R)+\varkappa\left(B R^{2}\right) B}{\lambda_{k 0}^{2}(0, R)}-B R^{2}\left(1-2 \alpha(k+1)^{-2 / 3}+\left(1-\varkappa\left(B R^{2}\right)\right)(k+1)^{-1}\right)\right. \\
& \left.-\frac{B^{2} R^{4}}{4(k+1)}\right]-2 B R^{2}\left[-1+2(\gamma+\log R)\left(1-\varkappa\left(B R^{2}\right)\right)-4 \alpha \zeta\left(\frac{2}{3}\right)\right]-B^{2} R^{4}(1+\gamma+\log R) .
\end{aligned}
$$

\section{Contribution of all eigenmodes with the effective "mass" for quasizero modes}

The desired zeta function corresponding to the one-loop correction with the effective "mass" for quasizero modes is written as

$$
\zeta_{\varkappa}^{\mathrm{gl}}(s)=\zeta^{\mathrm{gl}}(s)-\zeta^{\mathrm{gl}(0)}(s)+\zeta_{\varkappa}^{\mathrm{gl}(0)}(s)
$$

The improvement due to the effective "mass" is accumulated in the last two terms given by Eqs. (A2) and (A3). The corresponding effective potential is given by (the color factor of $\sqrt{3} / 2$ is restored)

$$
\begin{aligned}
\delta U_{\varkappa}^{\mathrm{gl}}(B, R)= & -\left.\frac{1}{2} \frac{d}{d s} \zeta_{\varkappa}^{\mathrm{gl}}(s)\right|_{s=0} \\
= & 4 \sum_{k=1}^{\infty} k\left[\sum_{m=-\frac{k-1}{2}}^{\frac{k-1}{2}} \Psi \mathrm{gl}\left(k, m, 0, \frac{\sqrt{3} B}{2}, R\right)-\frac{3}{4} B^{2} R^{4} \frac{1}{24}\left(1-\frac{1}{k}-\frac{5}{k^{2}}\right)\right] \\
& -\frac{B^{2} R^{4}}{48}(31+30 \gamma-30 \log 2+30 \log R)+\frac{3 B^{4} R^{8}}{5120} \\
& +4 \sum_{k=0}^{\infty}\left[(k+1) \log \frac{\lambda_{\uparrow k \frac{k}{2} 0}^{2}(B, R)+\varkappa\left(B R^{2}\right) B}{\lambda_{k \frac{k}{2}}^{2}(B, R)}+\frac{\sqrt{3}}{2} B R^{2} \varkappa\left(B R^{2}\right)(k+1)^{-1}\right]+2 \sqrt{3} B R^{2} \varkappa\left(B R^{2}\right)(\gamma+\log R) .
\end{aligned}
$$

Thus, the obtained effective potential is not an even function of $B$. To restore invariance under $B \rightarrow-B$, one adds the term $\varkappa$ to the contribution of modes $\lambda_{\downarrow k,-\frac{k}{2}, 0}^{2}(B, R)$ (these modes become quasizero when the field $B$ changes to the opposite direction). The function $\varkappa(z)$ should also be an even function (possibly constant). After these steps, one obtains Eq. (27) for the effective potential.

\section{APPENDIX B: QUARK EIGENMODES}

The equation for the eigenvalues of quark fields in the presence of a homogeneous (anti-)self-dual gluon field reads

$$
\not p \psi=\lambda \psi
$$

Here,

$$
\begin{aligned}
D_{\mu} & =\partial_{\mu}-i \hat{B}_{\mu}, \quad \hat{B}_{\mu}=\hat{n} B_{\mu}, B_{\mu}=-\frac{1}{2} B_{\mu \nu} x_{\nu}, \\
B_{\mu \nu} & = \pm \frac{1}{2} \varepsilon_{\mu \nu \alpha \beta} B_{\alpha \beta}, \\
\hat{n} & =\cos \xi t^{3}+\sin \xi t^{8}, \quad \xi=\frac{\pi}{6}+\frac{\pi}{3} k, \quad k=0,1, \ldots, 5,
\end{aligned}
$$

and $t_{i}$ are generators of $S U(3)$ in the fundamental representation. The field-strength tensor $B_{\mu \nu}$ may be parametrized as

$$
B_{i j}=\varepsilon_{i j k} B_{k}, \quad B_{i 4}= \pm B_{i}, \quad B=\sqrt{B_{1}^{2}+B_{2}^{2}+B_{3}^{2}}
$$

where "+" stands for a self-dual field and "-" for an anti-self-dual field. We choose the anti-Hermitian chiral representation of gamma matrices,

$$
\begin{aligned}
\left\{\gamma_{\mu}, \gamma_{\nu}\right\} & =-2 \delta_{\mu \nu}, \quad \gamma_{i}=\left(\begin{array}{cc}
0 & \sigma_{i} \\
-\sigma_{i} & 0
\end{array}\right), \\
\gamma_{4} & =i\left(\begin{array}{ll}
0 & \mathbf{1} \\
\mathbf{1} & 0
\end{array}\right), \quad \gamma_{5}=\gamma_{1} \gamma_{2} \gamma_{3} \gamma_{4}=\left(\begin{array}{cc}
\mathbf{1} & 0 \\
0 & -\mathbf{1}
\end{array}\right) .
\end{aligned}
$$

It is convenient to introduce the projectors

$$
\Sigma_{ \pm}=\frac{1}{2}\left(1 \pm \frac{\Sigma_{i} B_{i}}{B}\right), \quad P_{ \pm}=\frac{1}{2}\left(1 \pm \gamma_{5}\right)
$$

where 


$$
\begin{aligned}
\Sigma_{i} & =\frac{1}{2} \varepsilon_{i j k} \sigma_{j k}, & \sigma_{\mu \nu} & =-\frac{1}{2} \varepsilon_{\mu \nu \alpha \beta} \sigma_{\alpha \beta} \gamma_{5}, \\
\sigma_{i j} & =\varepsilon_{i j k} \Sigma_{k}, & \sigma_{i 4} & =-\gamma_{5} \Sigma_{i} .
\end{aligned}
$$

For a self-dual or anti-self-dual field $B_{\mu \nu}$, one obtains the identities

$$
\begin{aligned}
\sigma_{\mu \nu} B_{\mu \nu} & =4 B P_{\mp}\left(\Sigma_{+}-\Sigma_{-}\right), \\
\gamma_{\mu} B_{\mu \nu} x_{\nu} P_{\mp} & =-i \not \not B P_{\mp}\left(\Sigma_{+}-\Sigma_{-}\right),
\end{aligned}
$$

where the upper sign corresponds to a self-dual field and the lower sign corresponds to an anti-self-dual field. The color matrix $\hat{n}$ is diagonal, and for the sake of simplicity we keep the notation $\hat{n}$ for its diagonal elements.

Acting on Eq. (B1) with the projectors $P_{ \pm}=\left(1 \pm \gamma_{5}\right) / 2$, one rewrites it as

$$
\begin{aligned}
& \not \supset P_{+} \psi=\lambda P_{-} \psi, \\
& \not D P_{-} \psi=\lambda P_{+} \psi,
\end{aligned}
$$

or

$$
\begin{aligned}
& \not D \psi_{+}=\lambda \psi_{-}, \\
& \not \psi_{-}=\lambda \psi_{+},
\end{aligned}
$$

where $\psi_{ \pm}=P_{ \pm} \psi$. Substituting one equation into the other (this is valid if $\lambda \neq 0$ ), one finds

$$
\not D^{2} \psi_{\mp}=\lambda^{2} \psi_{\mp},
$$

$$
\lambda \psi_{ \pm}=\not D \psi_{\mp},
$$

$$
\psi=\psi_{+}+\psi_{-}=\left(\frac{\not D}{\lambda}+1\right) \psi_{\mp}
$$

Only one chiral component is independent, and the other one is found via Eq. (B3). The expression for $\gamma_{\mu} B_{\mu \nu} x_{\nu} P_{ \pm}$ $\left(\gamma_{\mu} B_{\mu \nu} x_{\nu}\right.$ originates from $\left.\not D\right)$ is more complex than $\gamma_{\mu} B_{\mu \nu} x_{\nu} P_{\mp}$, so we find $\psi_{-}$from Eq. (B2) in the case of a self-dual field and $\psi_{+}$in the case of an anti-self-dual field. Here and below, if \pm or $\mp$ appears alongside the Dirac operator $\not D$ or its eigenmode $\psi$, the upper sign should be taken for a self-dual field and the lower sign for an anti-selfdual field. $\eta$ is the normal to the surface of a sphere, $\eta^{2}=1$.

The analogue of the total angular momentum in Euclidean space is [53]

$$
\begin{aligned}
J_{\mu \nu} & =K_{\mu \nu}+S_{\mu \nu}, \\
K_{\mu \nu} & =-i\left(x_{\mu} \partial_{\nu}-x_{\nu} \partial_{\mu}\right), S_{\mu \nu}=\frac{i}{4}\left[\gamma_{\mu}, \gamma_{\nu}\right], \\
J_{i}^{ \pm} & =\frac{1}{2}\left(\frac{1}{2} \varepsilon_{i j k} J_{j k} \pm J_{i 4}\right)=\frac{1}{2}\left(\frac{1}{2} \varepsilon_{i j k} K_{j k} \pm K_{i 4}\right)+\frac{1}{2}\left(\frac{1}{2} \varepsilon_{i j k} S_{j k} \pm S_{i 4}\right)=K_{i}^{ \pm}+\frac{1}{2} P_{\mp} \Sigma_{i} .
\end{aligned}
$$

The algebra of operators $K_{\mu \nu}, S_{\mu \nu}, J_{\mu \nu}$ splits into two $s o(3)$ algebras,

$$
\left[K_{i}^{+}, K_{j}^{+}\right]=i \varepsilon_{i j k} K_{k}^{+},\left[K_{i}^{-}, K_{j}^{-}\right]=i \varepsilon_{i j k} K_{k}^{-},\left[K_{i}^{+}, K_{j}^{-}\right]=0,
$$

and analogous relations for $J^{ \pm}$. One introduces lowering and raising operators,

$$
\begin{aligned}
\Sigma^{( \pm)} & =\frac{1}{2}\left(\Sigma_{1} \pm i \Sigma_{2}\right), \quad K^{( \pm)}=K_{1} \pm i K_{2}, \\
{\left[K_{3}, K^{( \pm)}\right] } & = \pm K^{( \pm)}, \quad\left[K^{(+)}, K^{(-)}\right]=2 K_{3} .
\end{aligned}
$$

We choose a reference frame such that the direction of the field coincides with the $z$ axis,

$$
B_{i}=\{0,0, B\} .
$$

Thus, Eq. (B2) can be cast into the form

$$
\begin{aligned}
& \left\{-\left[\frac{1}{r^{3}} \partial_{r} r^{3} \partial_{r}-\frac{4}{r^{2}} \mathbf{K}_{\mp}^{2}+2 \hat{B} K_{2,1}^{z}-\frac{1}{4} \hat{B}^{2} r^{2}\right]-2 \hat{B}\right\} \psi_{\mp+}=\lambda^{2} \psi_{\mp+}, \\
& \left\{-\left[\frac{1}{r^{3}} \partial_{r} r^{3} \partial_{r}-\frac{4}{r^{2}} \mathbf{K}_{\mp}^{2}+2 \hat{B} K_{2,1}^{z}-\frac{1}{4} \hat{B}^{2} r^{2}\right]+2 \hat{B}\right\} \psi_{\mp-}=\lambda^{2} \psi_{\mp-},
\end{aligned}
$$

where $c$ in $\psi_{c s}$ stands for chirality, and $s$ stands for spin. It is convenient to introduce basis spinors $u_{c s}$, 


$$
P_{ \pm} u_{ \pm s}=u_{ \pm s}, P_{ \pm} u_{\mp s}=0, \quad \Sigma_{ \pm} u_{c \pm}=u_{c \pm}, \quad \Sigma_{ \pm} u_{c \mp}=0, \quad u_{c s}^{\dagger} u_{c^{\prime} s^{\prime}}=\delta_{c c^{\prime}} \delta_{s s^{\prime}}, \quad \Sigma^{(\mp)} u_{c \pm}=u_{c \mp}, \Sigma^{( \pm)} u_{c \pm}=0 .
$$

The solutions are

$$
\begin{aligned}
\psi_{\mp+}^{k m_{1} m_{2}} & =\left(\frac{\hat{B} r^{2}}{2}\right)^{k / 2} e^{-\hat{B} r^{2} / 4} M\left(\frac{k}{2}+1-m_{2,1}+\frac{-2 \hat{B}-\lambda^{2}}{2 \hat{B}}, k+2, \frac{\hat{B} r^{2}}{2}\right) Y_{\frac{k}{2} m_{1} m_{2}}(\varphi, \chi, \eta) u_{\mp+}, \\
\psi_{\mp-}^{k m_{1} m_{2}} & =\left(\frac{\hat{B} r^{2}}{2}\right)^{k / 2} e^{-\hat{B} r^{2} / 4} M\left(\frac{k}{2}+1-m_{2,1}+\frac{2 \hat{B}-\lambda^{2}}{2 \hat{B}}, k+2, \frac{\hat{B} r^{2}}{2}\right) Y_{\frac{k}{2} m_{1} m_{2}}(\varphi, \chi, \eta) u_{\mp-},
\end{aligned}
$$

where $Y_{\frac{k}{2} m_{1} m_{2}}$ are spherical harmonics in four-dimensional Euclidean space [53].

The solutions of Eq. (B1) may be characterized by eigenvalues of independent operators $\mathbf{J}^{\mp 2}, J_{3}^{\mp}, J_{3}^{ \pm}, L$ (see Refs [54-56]). Here,

$$
L=i \gamma_{\mu} \gamma_{5} B_{\mu \nu} D_{\nu}
$$

and upper and lower signs should be taken for a self-dual field and an anti-self-dual field, respectively. The solutions to the eigenvalue problem (B1),

$$
\left(\frac{\not D}{\lambda}+1\right) \psi_{c s}^{k m_{1} m_{2}}
$$

where $\psi_{c s}^{k m_{1} m_{2}}(c=\mp, s= \pm)$ are solutions of Eq. (B2) given by Eqs. (B5) and (B6), diagonalize all of these operators.

Now, we impose the bag boundary condition (11). Substituting Eq. (B4) into Eq. (11) and acting on it with the operators $P_{ \pm}$, we find that the boundary condition reduces to

$$
i e^{-i \alpha \gamma_{5}} \not \frac{\not D}{\lambda} \psi_{\mp}(x)=\psi_{\mp}(x), \quad x^{2}=R^{2},
$$

where $\psi_{\mp}$ are solutions of Eq. (B2). The boundary condition breaks the symmetry associated with the generator $L$. In order to satisfy the boundary condition, we have to mix the eigenstates of the operator $L$.

Let us consider the case of a self-dual field (the case of an anti-self-dual field is obtained via $m_{2} \rightarrow m_{1}, \alpha \rightarrow-\alpha$ ). To satisfy the boundary condition, a linear combination of solutions with equal projection of total angular momenta $j_{3}$ is constructed. At a given $k$, there is one solution for the maximal value $j_{3}=\frac{k}{2}+\frac{1}{2}$, one solution for the minimal value $j_{3}=-\frac{k}{2}-\frac{1}{2}$, and two solutions for each intermediate $j_{3}=-\frac{k}{2}+\frac{1}{2}, \ldots, \frac{k}{2}-\frac{1}{2}$ (for $k>0$ ). Below we consider these three cases.

\section{Intermediate values of $\boldsymbol{j}_{3}$}

As described above, we consider the linear combination

$$
\psi_{-}=C_{1} f\left(k, m_{2}+1, r\right) Y_{\frac{k}{2}, m_{1}, m_{2}} u_{-+}+C_{2} f\left(k, m_{2}, r\right) Y_{\frac{k}{2}, m_{1}, m_{2}+1}(\varphi, \chi, \eta) u_{--}
$$

for

$$
m_{2}=j_{3}-\frac{1}{2}=-\frac{k}{2},-\frac{k}{2}+1, \ldots, \frac{k}{2}-1, \quad k=1,2, \ldots,
$$

where we introduced the notation

$$
f(k, m, r)=\left(\frac{\hat{B} r^{2}}{2}\right)^{k / 2} e^{-\hat{B} r^{2} / 4} M\left(\frac{k}{2}+1-m+\frac{-\lambda^{2}}{2 \hat{B}}, k+2, \frac{\hat{B} r^{2}}{2}\right) .
$$

The boundary condition reads

$$
\begin{aligned}
& \frac{1}{\lambda}\left\{C_{1}\left[-\partial_{r}-\frac{\hat{B} R}{2}+\frac{2}{R} m_{2}\right] f\left(k, m_{2}+1, R\right)+C_{2} \sqrt{\frac{k}{2}\left(\frac{k}{2}+1\right)-m_{2}\left(m_{2}+1\right)} \frac{2}{R} f\left(k, m_{2}, R\right)\right\}=-i e^{-i \alpha} C_{1} f\left(k, m_{2}+1, R\right), \\
& \frac{1}{\lambda}\left\{C_{2}\left[-\partial_{r}+\frac{\hat{B} R}{2}-\frac{2}{R}\left(m_{2}+1\right)\right] f\left(k, m_{2}, R\right)+C_{1} \sqrt{\frac{k}{2}\left(\frac{k}{2}+1\right)-m_{2}\left(m_{2}+1\right)} \frac{2}{R} f\left(k, m_{2}+1, R\right)\right\}=-i e^{-i \alpha} C_{2} f\left(k, m_{2}, R\right) .
\end{aligned}
$$


Using the identities (see Eqs. 13.4.10 and 13.4.11 of Ref. [57])

$$
\begin{aligned}
a M(a+1, b, z) & =a M(a, b, z)+z M^{\prime}(a, b, z), \\
(b-a) M(a-1, b, z) & =(b-a-z) M(a, b, z)+z M^{\prime}(a, b, z),
\end{aligned}
$$

we bring these equations to the form

$$
\begin{aligned}
& \frac{C_{1}}{\lambda}\left[\left(-\frac{\lambda^{2}}{2 \hat{B}}+i e^{-i \alpha} \frac{\lambda R}{2}\right) M\left(\frac{k}{2}-m_{2}+\frac{-\lambda^{2}}{2 \hat{B}}, k+2, \frac{\hat{B} R^{2}}{2}\right)-\left(\frac{k}{2}-m_{2}+\frac{-\lambda^{2}}{2 \hat{B}}\right) M\left(\frac{k}{2}+1-m_{2}+\frac{-\lambda^{2}}{2 \hat{B}}, k+2, \frac{\hat{B} R^{2}}{2}\right)\right] \\
& \quad+\frac{C_{2}}{\lambda} \sqrt{\frac{k}{2}\left(\frac{k}{2}+1\right)-m_{2}\left(m_{2}+1\right)} M\left(\frac{k}{2}+1-m_{2}+\frac{-\lambda^{2}}{2 \hat{B}}, k+2, \frac{\hat{B} R^{2}}{2}\right)=0, \\
& \frac{C_{2}}{\lambda}\left[\left(\frac{\lambda^{2}}{2 \hat{B}}+i e^{-i \alpha} \frac{\lambda R}{2}\right) M\left(\frac{k}{2}+1-m_{2}+\frac{-\lambda^{2}}{2 \hat{B}}, k+2, \frac{\hat{B} R^{2}}{2}\right)-\left(\frac{k}{2}+1+m_{2}+\frac{\lambda^{2}}{2 \hat{B}}\right) M\left(\frac{k}{2}-m_{2}+\frac{-\lambda^{2}}{2 \hat{B}}, k+2, \frac{\hat{B} R^{2}}{2}\right)\right] \\
& \quad+\frac{C_{1}}{\lambda} \sqrt{\frac{k}{2}\left(\frac{k}{2}+1\right)-m_{2}\left(m_{2}+1\right)} M\left(\frac{k}{2}-m_{2}+\frac{-\lambda^{2}}{2 \hat{B}}, k+2, \frac{\hat{B} R^{2}}{2}\right)=0 .
\end{aligned}
$$

This system is uniform with respect to $C_{1}, C_{2}$ and has a nontrivial solution if the determinant of the system is zero. The latter requirement leads to the equation for eigenvalues:

$$
\begin{aligned}
\frac{1}{\lambda^{2}} & {\left[\left(-\frac{\lambda^{2}}{2 \hat{B}}+i e^{-i \alpha} \frac{\lambda R}{2}\right) M\left(\frac{k}{2}+\frac{1}{2}-j_{3}+\frac{-\lambda^{2}}{2 \hat{B}}, k+2, \frac{\hat{B} R^{2}}{2}\right)-\left(\frac{k}{2}+\frac{1}{2}-j_{3}+\frac{-\lambda^{2}}{2 \hat{B}}\right) M\left(\frac{k}{2}+\frac{3}{2}-j_{3}+\frac{-\lambda^{2}}{2 \hat{B}}, k+2, \frac{\hat{B} R^{2}}{2}\right)\right] } \\
\times & {\left[\left(\frac{\lambda^{2}}{2 \hat{B}}+i e^{-i \alpha} \frac{\lambda R}{2}\right) M\left(\frac{k}{2}+\frac{3}{2}-j_{3}+\frac{-\lambda^{2}}{2 \hat{B}}, k+2, \frac{\hat{B} R^{2}}{2}\right)-\left(\frac{k}{2}+\frac{1}{2}+j_{3}+\frac{\lambda^{2}}{2 \hat{B}}\right) M\left(\frac{k}{2}+\frac{1}{2}-j_{3}+\frac{-\lambda^{2}}{2 \hat{B}}, k+2, \frac{\hat{B} R^{2}}{2}\right)\right] } \\
& -\frac{1}{\lambda^{2}}\left[\frac{k}{2}\left(\frac{k}{2}+1\right)-j_{3}^{2}+\frac{1}{4}\right] M\left(\frac{k}{2}+\frac{1}{2}-j_{3}+\frac{-\lambda^{2}}{2 \hat{B}}, k+2, \frac{\hat{B} R^{2}}{2}\right) M\left(\frac{k}{2}+\frac{3}{2}-j_{3}+\frac{-\lambda^{2}}{2 \hat{B}}, k+2, \frac{\hat{B} R^{2}}{2}\right)=0,
\end{aligned}
$$

where the substitution $m_{2} \rightarrow j_{3}-\frac{1}{2}$ was made.

\section{Maximal value of $\boldsymbol{j}_{3}$}

In this case, there is only one function with $j_{3}=\frac{k}{2}+\frac{1}{2}$ at a given $k=0,1,2, \ldots$,

$$
\psi_{-}=C_{1} f\left(k, m_{2}+1, r\right) Y_{\frac{k}{2}, m_{1}, m_{2}} u_{-+}, \quad m_{2}=j_{3}-\frac{1}{2}=\frac{k}{2} .
$$

The boundary condition in this case can be obtained from Eq. (B7) with $C_{2}=0$. After simplification, we find

$$
M\left(-\frac{\lambda^{2}}{2 \hat{B}}, k+2, \frac{\hat{B} R^{2}}{2}\right)-i e^{i \alpha} \frac{\lambda R}{2(k+2)} M\left(1-\frac{\lambda^{2}}{2 \hat{B}}, k+3, \frac{\hat{B} R^{2}}{2}\right)=0 .
$$

\section{Minimal value of $\boldsymbol{j}_{3}$}

There is also only one function with $j_{3}=-\frac{k}{2}-\frac{1}{2}$ at a given $k=0,1,2, \ldots$,

$$
\psi_{-}=C_{2} f\left(k, m_{2}, r\right) Y_{\frac{k}{2}, m_{1}, m_{2}+1}(\varphi, \chi, \eta) u_{--}, m_{2}=j_{3}-\frac{1}{2}=-\frac{k}{2}-1 .
$$

The boundary condition can be obtained from Eq. (B8) with $C_{1}=0$. After simplification and Kummer transformation (see Eq. 13.1.27 of Ref. [57]), 


$$
M(a, b, z)=e^{z} M(b-a, b,-z),
$$

we obtain

$$
M\left(\frac{\lambda^{2}}{2 \hat{B}}, k+2,-\frac{\hat{B} R^{2}}{2}\right)-i e^{i \alpha} \frac{\lambda R}{2(k+2)} M\left(1+\frac{\lambda^{2}}{2 \hat{B}}, k+3,-\frac{\hat{B} R^{2}}{2}\right)=0 .
$$

\section{APPENDIX C: ZETA FUNCTION FOR QUARK FIELDS}

We put the chiral angle $\alpha=\pi / 2$, so the Dirac operator is Hermitian and eigenvalues are real. The zeta function $\zeta(s)$ of fermions is split into two parts:

$$
\begin{aligned}
\zeta^{\mathrm{q}}(s) & =\cos (\pi s) \zeta_{\not p^{2}}\left(\frac{s}{2}\right)-i \sin (\pi s) \eta(s), \\
\zeta_{\not p^{2}}(s) & =\operatorname{Tr} \sum_{k, j_{3}, n}(k+1)\left(\frac{1}{\left|\lambda_{k j_{3} n}(\hat{B}, R)\right|^{2 s}}-\frac{1}{\left|\lambda_{k j_{3} n}(0, R)\right|^{2 s}}\right), \\
\eta(s) & =\operatorname{Tr} \sum_{k, j_{3}, n}(k+1)\left(\frac{\operatorname{sign} \lambda_{k j_{3} n}(\hat{B}, R)}{\left|\lambda_{k j_{3} n}(\hat{B}, R)\right|^{s}}-\frac{\operatorname{sign} \lambda_{k j_{3} n}(0, R)}{\left|\lambda_{k j_{3} n}(0, R)\right|^{s}}\right) .
\end{aligned}
$$

The eigenvalues $\lambda_{k j n}$ are found from the equations $A\left(\lambda, k, j_{3}, \hat{B}, R \mid \alpha\right)=0$. The set of eigenvalues for $j_{3}= \pm \frac{k+1}{2}$ is determined by the equations

$$
\begin{gathered}
A\left(\lambda, k, \frac{k+1}{2}, \hat{B}, R\right)=M\left(-\frac{\lambda^{2}}{2 \hat{B}}, k+2, \frac{\hat{B} R^{2}}{2}\right)+\frac{R \lambda}{2(k+2)} M\left(1-\frac{\lambda^{2}}{2 \hat{B}}, k+3, \frac{\hat{B} R^{2}}{2}\right)=0, \\
A\left(\lambda, k,-\frac{k+1}{2}, \hat{B}, R\right)=M\left(\frac{\lambda^{2}}{2 \hat{B}}, k+2,-\frac{\hat{B} R^{2}}{2}\right)+\frac{R \lambda}{2(k+2)} M\left(1+\frac{\lambda^{2}}{2 \hat{B}}, k+3,-\frac{\hat{B} R^{2}}{2}\right)=0 .
\end{gathered}
$$

At $B=0$ the equations take the form

$$
(k+1) !\left(\frac{\lambda R}{2}\right)^{-k-1}\left[J_{k+1}(\lambda R)+J_{k+2}(\lambda R)\right]=0 .
$$

The eigenvalues for $j_{3}=-\frac{k-1}{2}, \ldots, \frac{k-1}{2}, k \geq 1$ are found from the equation

$$
\begin{aligned}
A\left(\lambda, k, j_{3}, \hat{B}, R\right)= & \exp \left(-\frac{\hat{B} R^{2}}{2}\right) \frac{1}{\lambda^{2}}\left[\frac{\lambda R}{2} M\left(\frac{k}{2}+\frac{1}{2}-j_{3}+\frac{-\lambda^{2}}{2 \hat{B}}, k+2, \frac{\hat{B} R^{2}}{2}\right)\right. \\
& \left.-\left(\frac{k}{2}+\frac{1}{2}-j_{3}\right) M\left(\frac{k}{2}+\frac{3}{2}-j_{3}+\frac{-\lambda^{2}}{2 \hat{B}}, k+2, \frac{\hat{B} R^{2}}{2}\right)+\frac{\lambda^{2} R^{2}}{4(k+2)} M\left(\frac{k}{2}+\frac{3}{2}-j_{3}+\frac{-\lambda^{2}}{2 \hat{B}}, k+3, \frac{\hat{B} R^{2}}{2}\right)\right] \\
& \times\left[\frac{\lambda R}{2} M\left(\frac{k}{2}+\frac{3}{2}-j_{3}+\frac{-\lambda^{2}}{2 \hat{B}}, k+2, \frac{\hat{B} R^{2}}{2}\right)\right. \\
& \left.-\left(\frac{k}{2}+\frac{1}{2}+j_{3}\right) M\left(\frac{k}{2}+\frac{1}{2}-j_{3}+\frac{-\lambda^{2}}{2 \hat{B}}, k+2, \frac{\hat{B} R^{2}}{2}\right)+\frac{\lambda^{2} R^{2}}{4(k+2)} M\left(\frac{k}{2}+\frac{3}{2}-j_{3}+\frac{-\lambda^{2}}{2 \hat{B}}, k+3, \frac{\hat{B} R^{2}}{2}\right)\right] \\
& -\exp \left(-\frac{\hat{B} R^{2}}{2}\right) \frac{1}{\lambda^{2}}\left[\frac{k}{2}\left(\frac{k}{2}+1\right)-j_{3}^{2}+\frac{1}{4}\right] \\
& \times M\left(\frac{k}{2}+\frac{1}{2}-j_{3}+\frac{-\lambda^{2}}{2 \hat{B}}, k+2, \frac{\hat{B} R^{2}}{2}\right) M\left(\frac{k}{2}+\frac{3}{2}-j_{3}+\frac{-\lambda^{2}}{2 \hat{B}}, k+2, \frac{\hat{B} R^{2}}{2}\right)=0 .
\end{aligned}
$$


Note the factor $\exp \left(-\frac{\hat{B} R^{2}}{2}\right)$ which does not affect the solutions of the equation, but makes the equation invariant with respect to $j_{3} \rightarrow-j_{3}, B \rightarrow-B$ (via Kummer transformation). So, the decomposition of $\zeta(s)$ and the free energy will contain only even powers of $B$. At $B \rightarrow 0$ this equation transforms to

$$
\begin{aligned}
A\left(\lambda, k, j_{3}, 0, R \mid \alpha\right)= & \frac{1}{\lambda^{2}}(k+1) !\left(\frac{\lambda R}{2}\right)^{-k-1}\left[\frac{\lambda R}{2} J_{k+1}(\lambda R)-\left(\frac{k}{2}+\frac{1}{2}-j\right) J_{k+1}(\lambda R)+\frac{\lambda R}{2} J_{k+2}(\lambda R)\right] \\
& \times(k+1) !\left(\frac{\lambda R}{2}\right)^{-k-1}\left[\frac{\lambda R}{2} J_{k+1}(\lambda R)-\left(\frac{k}{2}+\frac{1}{2}+j\right) J_{k+1}(\lambda R)+\frac{\lambda R}{2} J_{k+2}(\lambda R)\right] \\
& -\frac{1}{\lambda^{2}}\left[\frac{k}{2}\left(\frac{k}{2}+1\right)-j^{2}+\frac{1}{4}\right]\left[(k+1) !\left(\frac{\lambda R}{2}\right)^{-k-1} J_{k+1}(\lambda R)\right]^{2}=0 .
\end{aligned}
$$

It follows that

$$
\zeta_{\not p^{2}}(s)=\zeta_{\not p^{2}}^{(1)}(s)+\zeta_{\not p^{2}}^{(2)}(s)
$$

where the first term summarizes contributions of the eigenvalues with maximal and minimal projections of total angular momentum $j_{3}= \pm \frac{k+1}{2}$, and the second term with intermediate values $j_{3}=-\frac{k-1}{2}, \ldots, \frac{k-1}{2}$. It is easily seen that

$$
A\left(\lambda, k, j_{3}, \hat{B}, R\right)=A\left(\lambda, k,-j_{3},-\hat{B}, R\right),
$$

so the invariance of the spectrum under the transformation $B \rightarrow-B$ is manifest.

\section{Contribution of maximal and minimal $j_{3}$}

We start with the representation

$$
\begin{aligned}
\zeta_{\not b^{2}}^{(1)}(s)= & \operatorname{Tr}\left\{\frac{\sin \pi s}{\pi} \sum_{k=0}^{\infty} \sum_{j_{3}= \pm \frac{k+1}{2}}(k+1) \int_{0}^{\infty} \frac{d t}{t^{2 s}} \frac{d}{d t} \Psi^{\mathrm{q}}\left(k+1, j_{3}, t, \hat{B}, R\right)\right\} \\
= & \operatorname{Tr}\left\{\frac{\sin \pi s}{\pi} \sum_{k=1}^{\infty} k^{1-2 s} \int_{0}^{\infty} \frac{d t}{t^{2 s}} \frac{d}{d t}\left[\sum_{j_{3}= \pm \frac{k}{2}} \Psi^{\mathrm{q}}\left(k, j_{3}, k t, \hat{B}, R\right)-\sum_{i=1}^{2} \frac{u_{i}^{\mathrm{q}}(t, \hat{B}, R)}{k^{i}}\right]\right. \\
& \left.+\frac{\sin \pi s}{\pi} \sum_{k=1}^{\infty} k^{1-2 s} \int_{0}^{\infty} \frac{d t}{t^{2 s}} \frac{d}{d t} \sum_{i=1}^{2} \frac{u_{i}^{\mathrm{q}}(t, \hat{B}, R)}{k^{i}}\right\} .
\end{aligned}
$$

We need a suitable asymptotic expansion for the hypergeometric function $M$ to find explicit expressions for counterterms $u_{i}^{\mathrm{q}}$. The expansion at large $k$ and fixed $j / k$ can be found with the help of the method described in Ref. [58] (Chapter 10, $\$$ ). We obtain

$$
\begin{aligned}
& M\left(\frac{m+1}{2}+\frac{k}{2}-j+n+\frac{k^{2} t^{2}}{4}, 1+k+m, z\right) \\
& \quad \sim \frac{2^{k+m} \Gamma(k+m+1)}{k^{k+m} \sqrt{2 \pi k}\left(1+t^{2} z\right)^{\frac{1}{4}}} \exp \left(k \sqrt{1+t^{2} z}-(k+m) \log \left[\sqrt{1+t^{2} z}+1\right]-\frac{2 j}{k} \frac{z}{\sqrt{1+t^{2} z}+1}+\frac{z}{2}\right) \sum_{i=0}^{\infty} \frac{A_{i}(z)}{k^{i}}
\end{aligned}
$$

for a fixed $z \geq 0$ and arbitrary constants $m, n$. The coefficients $A_{i}(z)$ are found with the help of the recursion relation $\left(i \geq 0, A_{0}=1\right)$

$$
A_{i+1}(z)=-\frac{1}{2} \phi-\frac{1}{2} \frac{d A_{i}}{d z} \frac{d z}{d \xi}+\int d z \frac{d \xi}{d z}\left(\psi+\frac{1}{2} \frac{d \phi}{d z} \frac{d z}{d \xi}-\frac{1}{4} \phi^{2}\right) A_{i}
$$

where 


$$
\frac{d \xi}{d z}=\frac{1}{2 z} \sqrt{1+t^{2} z}, \quad \phi=\frac{2}{1+t^{2} z}\left(m-\frac{2 j}{k}\right), \quad \psi=\frac{m^{2}+4 n z}{1+t^{2} z}+\frac{4 t^{2} z+4 z^{2}-t^{4} z^{2}+8 t^{2} z^{3}+4 t^{4} z^{4}}{\left(1+t^{2} z\right)^{3}} .
$$

The constants of integration are fixed by the requirement

$$
\lim _{t \rightarrow \infty} A_{i}(z)=0, i \geq 1
$$

The asymptotic expansion for the modified Bessel function $I$ is given by (see Eqs. 10.41.3, 10.41.7, and 10.41.9 of Ref. [59])

$$
I_{k}(k t)=\frac{1}{\sqrt{2 \pi k}\left(1+t^{2}\right)^{\frac{1}{4}}} \exp \left(k \sqrt{1+t^{2}}+k \log \frac{t}{1+\sqrt{1+t^{2}}}\right) \sum_{i=0}^{\infty} \frac{U_{i}(p)}{k^{i}},
$$

where

$$
U_{k+1}(p)=\frac{1}{2} p^{2}\left(1-p^{2}\right) U_{k}^{\prime}(p)+\frac{1}{8} \int_{0}^{p} d p^{\prime}\left(1-5 p^{\prime 2}\right) U_{k}\left(p^{\prime}\right), \quad p=\sqrt{1+t^{2}} .
$$

Now, we substitute Eqs. (C1) and (C2) into $\Psi^{\mathrm{q}}$, expand it in powers of $k^{-1}$, sum over $m$, and compute the derivative with respect to $t$. The functions $u_{i}^{\mathrm{q}}$ are given by

$$
\begin{aligned}
& u_{1}^{\mathrm{q}}(t, \hat{B}, R)=\hat{B}^{2} R^{4} \frac{-8-4 R^{2} t^{2}+R^{4} t^{4}+8 \sqrt{1+R^{2} t^{2}}}{6 R^{4} t^{4} \sqrt{1+R^{2} t^{2}}} \\
& u_{2}^{\mathrm{q}}(t, \hat{B}, R)=\hat{B}^{2} R^{4} \frac{-8-12 R^{2} t^{2}-3 R^{4} t^{4}+8 \sqrt{1+R^{2} t^{2}}+8 R^{2} t^{2} \sqrt{1+R^{2} t^{2}}}{4 R^{4} t^{4}\left(1+R^{2} t^{2}\right)^{\frac{3}{2}}} .
\end{aligned}
$$

Evaluating the sums and integrals, one finds

$$
\sum_{k=1}^{\infty} k^{1-2 s} \int_{0}^{\infty} \frac{d t}{t^{2 s}} \frac{d}{d t} \sum_{i=1}^{2} \frac{u_{i}^{\mathrm{q}}(t, \hat{B}, R)}{k^{i}}=\hat{B}^{2} R^{4+2 s}\left\{\zeta(2 s) \frac{s \Gamma(1-s) \Gamma\left(s+\frac{1}{2}\right)}{2 \sqrt{\pi}(s+2)}-\zeta(1+2 s) \frac{s \Gamma(1-s) \Gamma\left(s+\frac{3}{2}\right)}{2 \sqrt{\pi}(s+2)}\right\} .
$$

The expansion of the counterterm in powers of $s$ is

$$
\frac{\sin \pi s}{\pi} \sum_{k=1}^{\infty} k^{1-2 s} \int_{0}^{\infty} \frac{d t}{t^{2 s}} \frac{d}{d t} \sum_{i=0}^{2} \frac{u_{i}^{\mathrm{q}}(t, \hat{B}, R)}{k^{i}}=-\frac{1}{16} \hat{B}^{2} R^{4} s+O\left(s^{2}\right),
$$

and the corresponding contribution to the effective potential reads

$$
\left.\frac{1}{2} \frac{d}{d s} \zeta_{\not p^{2}}^{(1)}(s)\right|_{s=0}=-\frac{1}{32} \operatorname{Tr} \hat{B}^{2} R^{4}=-\frac{1}{64} B^{2} R^{4}
$$




\section{Contribution of intermediate $j_{3}$}

The starting expression in this case is

$$
\begin{aligned}
\zeta_{\not p^{2}}^{(2)}(s)= & \operatorname{Tr}\left\{\frac{\sin \pi s}{\pi} \sum_{k=1}^{\infty} \sum_{j_{3}=-\frac{k-1}{2}}^{\frac{k-1}{2}}(k+1) \int_{0}^{\infty} \frac{d t}{t^{2 s}} \frac{d}{d t} \Psi^{\mathrm{q}}\left(k+1, j_{3}, t, \hat{B}, R\right)\right\} \\
= & \operatorname{Tr}\left\{\frac{\sin \pi s}{\pi} \sum_{k=1}^{\infty}(k+1) k^{-2 s} \int_{0}^{\infty} \frac{d t}{t^{2 s}} \frac{d}{d t}\left[\sum_{j_{3}=-\frac{k-1}{2}}^{\frac{k-1}{2}} \Psi^{\mathrm{q}}\left(k+1, j_{3}, k t, \hat{B}, R\right)-\sum_{i=0}^{2} \frac{u_{i}^{\mathrm{q}}(t, \hat{B}, R)}{k^{i}}\right]\right. \\
& \left.+\frac{\sin \pi s}{\pi} \sum_{k=1}^{\infty}(k+1) k^{-2 s} \int_{0}^{\infty} \frac{d t}{t^{2 s}} \frac{d}{d t} \sum_{i=0}^{2} \frac{u_{i}^{\mathrm{q}}(t, \hat{B}, R)}{k^{i}}\right\},
\end{aligned}
$$

where

$$
\begin{aligned}
u_{0}^{\mathrm{q}}(t, B, R)= & \hat{B}^{2} R^{4} \frac{R^{4} t^{4}-2 R^{2} t^{2}-4+4\left(1+R^{2} t^{2}\right)^{1 / 2}}{6 R^{4} t^{4}\left(1+R^{2} t^{2}\right)^{1 / 2}}, \\
u_{1}^{\mathrm{q}}(t, \hat{B}, R)= & \hat{B}^{2} R^{4} \frac{-3 R^{4} t^{4}-8+8\left(1+R^{2} t^{2}\right)^{1 / 2}-12 R^{2} t^{2}+8 R^{2} t^{2}\left(1+R^{2} t^{2}\right)^{1 / 2}}{6 t^{4}\left(1+R^{2} t^{2}\right)^{\frac{3}{2}}}, \\
u_{2}^{\mathrm{q}}(t, \hat{B}, R)= & \frac{\hat{B}^{2} R^{4}}{48\left(1+R^{2} t^{2}\right)^{1 / 2}}\left[-\frac{32}{R^{2} t^{2}}+\frac{R^{2} t^{2}\left(8+13 R^{2} t^{2}\right)}{\left(1+R^{2} t^{2}\right)^{3}}-64 \frac{1-\left(1+R^{2} t^{2}\right)^{1 / 2}}{R^{4} t^{4}}\right] \\
& +\frac{\hat{B}^{4} R^{8}}{480\left(1+R^{2} t^{2}\right)^{1 / 2}}\left[-\frac{128}{R^{6} t^{6}}+\frac{32}{R^{4} t^{4}}-\frac{16}{R^{2} t^{2}}+\frac{10+13 R^{2} t^{2}}{\left(1+R^{2} t^{2}\right)^{2}}-256 \frac{1-\left(1+R^{2} t^{2}\right)^{1 / 2}}{R^{8} t^{8}}\right] .
\end{aligned}
$$

We find

$$
\begin{aligned}
\sum_{k=1}^{\infty}( & k+1) k^{-2 s} \int_{0}^{\infty} \frac{d t}{t^{2 s}} \frac{d}{d t} \sum_{i=0}^{2} \frac{u_{i}^{\mathrm{q}}(t, \hat{B}, R)}{k^{i}} \\
= & \hat{B}^{2} R^{4+2 s}\left\{\zeta(-1+2 s) \frac{-\Gamma(2-s) \Gamma(1 / 2+s)}{6 \sqrt{\pi}(2+s)}+\zeta(2 s) \frac{-\left(1+2 s^{2}\right) \Gamma(1-s) \Gamma(1 / 2+s)}{6 \sqrt{\pi}(2+s)}\right. \\
& \left.+\zeta(1+2 s) \frac{(12+s(4-s)(1+s)) \Gamma(1-s) \Gamma(3 / 2+s)}{18 \sqrt{\pi}(2+s)}\right\}-\hat{B}^{4} R^{8+2 s} \zeta(1+2 s) \frac{s \Gamma(2-s) \Gamma(3 / 2+s)}{240 \sqrt{\pi}(4+s)} .
\end{aligned}
$$

The small- $s$ expansion is

$$
\begin{aligned}
& \frac{\sin \pi s}{\pi} \sum_{k=1}^{\infty}(k+1) k^{-2 s} \int_{0}^{\infty} \frac{d t}{t^{2 s}} \frac{d}{d t} \sum_{i=0}^{2} \frac{u_{i}^{\mathrm{q}}(t, \hat{B}, R)}{k^{i}} \\
& \quad=\hat{B}^{2} R^{4}\left[\frac{1}{12}+\frac{1}{144}\left(29+24 \gamma-24 \log 2+4 \pi^{2}+24 \log R\right) s\right]+\hat{B}^{4} R^{8}\left[-\frac{1}{1920} s\right]+O\left(s^{2}\right) .
\end{aligned}
$$

And we find the corresponding contribution to the effective potentiall

$$
\begin{aligned}
\left.\frac{1}{2} \frac{d}{d s} \zeta_{\not b^{2}}^{(2)}(s)\right|_{s=0}= & -\frac{1}{2} \operatorname{Tr} \sum_{k=1}^{\infty}(k+1)\left[\sum_{j_{3}=-\frac{k-1}{2}}^{\frac{k-1}{2}} \Psi^{\mathrm{q}}\left(k, j_{3}, 0, \hat{B}, R\right)-\hat{B}^{2} R^{4} \frac{1}{12}\left(1-\frac{2}{k^{2}}\right)\right] \\
& +\frac{B^{2} R^{4}}{576}\left(29+24 \gamma-24 \log 2+4 \pi^{2}+24 \log R\right)-\frac{B^{4} R^{8}}{30720}
\end{aligned}
$$

Combining the two contributions $\zeta_{\not \not^{2}}^{(1)}$ and $\zeta_{\not \not^{2}}^{(2)}$ considered above, one obtains Eq. (30). 


\section{APPENDIX D: ASYMPTOTIC EXPANSION FOR QUASIZERO EIGENVALUES}

Let $x$ stand for the smallest solution of the equation. The equation

$$
M\left(-\frac{x(z)^{2}}{2 z}, k+2, \frac{z}{2}\right)=0, \quad k=0,1,2, \ldots
$$

reduces to

$$
\left(\frac{x(0)}{2}\right)^{-k-1} J_{k+1}(x(0))=0, \quad k=0,1, \ldots
$$

at $z \rightarrow 0$. The solution can be sought in the form of a power series in $z$,

$$
x(z)=\sum_{i=0}^{\infty} \frac{z^{i}}{i !} \frac{\partial^{i}}{\partial z^{i}} x^{(i)}(0) .
$$

The derivatives $x^{(i)}(0)$ can be found successively from the equations

$$
\left.\frac{\partial^{i}}{\partial z^{i}} M\left(-\frac{x(z)^{2}}{2 z}, k+2, \frac{z}{2}\right)\right|_{z=0}=0 .
$$

We find

$$
\begin{aligned}
\left.\frac{d x(z)}{d z}\right|_{z=0} & =-\frac{k+2}{2 x(0)}, \\
\left.\frac{d^{2} x(z)}{d z^{2}}\right|_{z=0} & =-\frac{k^{2}+8 k+12-x^{2}(0)}{12 x^{3}(0)}, \\
\left.\frac{d^{3} x(z)}{d z^{3}}\right|_{z=0} & =-\frac{(k+2)\left(k^{2}+8 k+12-x^{2}(0)\right)}{8 x^{5}(0)},
\end{aligned}
$$

where we used the identities (see Eq. 9.1.27 of Ref. [57])

$$
\begin{aligned}
J_{\nu-1}(z)+J_{\nu+1} & =\frac{2 \nu}{z} J_{\nu}(z), \\
J_{\nu}^{\prime}(z) & =-J_{\nu+1}(z)+\frac{\nu}{z} J_{\nu}(z)
\end{aligned}
$$

and the fact that

$$
J_{k+1}(x(0))=0 .
$$

Next, we use the uniform asymptotic expansion of zeros of the Bessel functions $J_{\nu}(z)$ (see Eq. 10.21.vii of Ref. [57]),

$\rho_{\nu}(t)=\nu \sum_{k=0}^{\infty} \frac{\alpha_{k}}{\nu^{2 k / 3}}, \theta\left(-2^{\frac{1}{3}} \alpha\right)=\pi t, \alpha_{0}=1$,

$$
\alpha_{1}=\alpha, \quad \alpha_{2}=\frac{3}{10} \alpha^{2}, \ldots,
$$

where $\theta(x)$ is the phase of Airy functions,

$$
\theta(z)=\arctan \frac{\operatorname{Ai}(z)}{\operatorname{Bi}(z)} .
$$

Quasizero solutions of Eq. (D1) at $z \rightarrow 0$ become the smallest zeroes of the Bessel functions that correspond to $t=1$. With $t=1$, Eq. (D3) gives the desired expansion for the first zero of $J_{k+1}(z)$,

$$
x(0)=(k+1) \sum_{i=0}^{\infty} \frac{\alpha_{k}}{(k+1)^{2 i / 3}},
$$

where $\alpha \approx 1.855757$. This series can be once again expanded in powers of $k$.

According to Eq. (D4), $x(0) \sim k$ for $k \gg 1$. One notices that the power of the leading-asymptotics term of the derivative $x^{(i)}(0)$ is smaller for larger $i$,

$$
x(0) \sim k,\left.\quad \frac{d x(a)}{d a}\right|_{a=0} \sim k^{0}, \ldots
$$

so we need only several terms in the series (D2) to find the asymptotic expansion of $x(z)$ in $k$ up to a given order:

$$
\begin{aligned}
x(z)= & k+\alpha k^{1 / 3}+1+\frac{3 \alpha^{2}}{10} k^{-1 / 3}+\frac{\alpha}{3} k^{-2 / 3} \\
& +\left(\frac{1}{70}-\frac{\alpha^{3}}{350}\right) k^{-1}+z\left[-\frac{1}{2}+\frac{\alpha}{2} k^{-2 / 3}-\frac{1}{2} k^{-1}\right] \\
& +O\left(k^{-4 / 3}\right) .
\end{aligned}
$$


[1] C. G. Callan, Jr., R. F. Dashen, and D. J. Gross, Toward a theory of the strong interactions, Phys. Rev. D 17, 2717 (1978).

[2] D. Diakonov and V. Yu. Petrov, Instanton based vacuum from Feynman variational principle, Nucl. Phys. B245, 259 (1984).

[3] A. Eichhorn, H. Gies, and J. M. Pawlowski, Gluon condensation and scaling exponents for the propagators in Yang-Mills theory, Phys. Rev. D 83, 045014 (2011); Erratum, Phys. Rev. D 83, 069903 (2011).

[4] G. K. Savvidy, Infrared instability of the vacuum state of gauge theories and asymptotic freedom, Phys. Lett. 71B, 133 (1977).

[5] H. Pagels and E. Tomboulis, Vacuum of the quantum YangMills theory and magnetostatics, Nucl. Phys. B143, 485 (1978).

[6] P. Minkowski, Comment on an incorrect interdependence of the PCAC_-quark masses, the potential $C P$ violating phase in QCD and the mass of the alleged axion, Phys. Lett. 76B, 439 (1978).

[7] H. Leutwyler, Vacuum fluctuations surrounding soft gluon fields, Phys. Lett. 96B, 154 (1980).

[8] H. Leutwyler, Constant gauge fields and their quantum fluctuations, Nucl. Phys. B179, 129 (1981).

[9] H. D. Trottier and R. M. Woloshyn, The Savvidy 'Ferromagnetic Vacuum' in Three-Dimensional Lattice Gauge Theory, Phys. Rev. Lett. 70, 2053 (1993).

[10] C. A. Flory, Covariant constant chromomagnetic fields and elimination of the one loop instabilities, Phys. Rev. D, 1983 (to be published).

[11] C. A. Flory, A selfdual gauge field, its quantum fluctuations, and interacting fermions, Phys. Rev. D 28, 1425 (1983).

[12] E. Elizalde and J. Soto, Zeta regularized Lagrangians for massive quarks in constant background mean fields, Ann. Phys. (N.Y.) 162, 192 (1985).

[13] G. Savvidy, From Heisenberg-Euler Lagrangian to the discovery of chromomagnetic gluon condensation, Eur. Phys. J. C 80, 165 (2020).

[14] S. N. Nedelko and V.E. Voronin, Domain wall network as QCD vacuum and the chromomagnetic trap formation under extreme conditions, Eur. Phys. J. A 51, 45 (2015).

[15] P. de Forcrand, Localization properties of fermions and bosons, AIP Conf. Proc. 892, 29 (2007).

[16] P. Moran and D. Leinweber, Buried treasure in the sand of the QCD vacuum, in QCD Downunder II, arXiv:0805.4246.

[17] F. Bruckmann, F. Gruber, N. Cundy, A. Schafer, and T. Lippert, Topology of dynamical lattice configurations including results from dynamical overlap fermions, Phys. Lett. B 707, 278 (2012).

[18] B. V. Galilo and S. N. Nedelko, Weyl group, $C P$ and the kink-like field configurations in the effective $\mathrm{SU}(3)$ gauge theory, Phys. Part. Nucl. Lett. 8, 67 (2011).

[19] D. P. George, A. Ram, J. E. Thompson, and R. R. Volkas, Symmetry breaking, subgroup embeddings and the Weyl group, Phys. Rev. D 87, 105009 (2013).

[20] V. A. Novikov, M. A. Shifman, A. I. Vainshtein, and V. I. Zakharov, Wilson's operator expansion: Can it fail?, Nucl. Phys. B249, 445 (1985).
[21] F. David, On the ambiguity of composite operators, IR renormalons and the status of the operator product expansion, Nucl. Phys. B234, 237 (1984).

[22] M. Shifman, New and old about renormalons: In memoriam Kolya Uraltsev, Int. J. Mod. Phys. A 30, 1543001 (2015).

[23] G. V. Efimov and S. N. Nedelko, Nambu-Jona-Lasinio model with the homogeneous background gluon field, Phys. Rev. D 51, 176 (1995).

[24] J. V. Burdanov, G. V. Efimov, S. N. Nedelko, and S. A. Solunin, Meson masses within the model of induced nonlocal quark currents, Phys. Rev. D 54, 4483 (1996).

[25] A. C. Kalloniatis and S. N. Nedelko, Confinement and chiral symmetry breaking via domain-like structures in the QCD vacuum, Phys. Rev. D 64, 114025 (2001).

[26] A. C. Kalloniatis and S. N. Nedelko, Realization of chiral symmetry in the domain model of QCD, Phys. Rev. D 69, 074029 (2004); Phys. Rev. DErratum, 70, 119903 (2004).

[27] S. N. Nedelko and V. E. Voronin, Regge spectra of excited mesons, harmonic confinement and QCD vacuum structure, Phys. Rev. D 93, 094010 (2016).

[28] S. N. Nedelko and V. E. Voronin, Influence of confining gluon configurations on the $P \rightarrow \gamma^{*} \gamma$ transition form factors, Phys. Rev. D 95, 074038 (2017).

[29] G. Bali, F. Bruckmann, G. Endrodi, Z. Fodor, S. Katz, S. Krieg, A. Schafer, and K. Szabo, The QCD phase diagram for external magnetic fields, J. High Energy Phys. 02 (2012) 044.

[30] G. Bali, F. Bruckmann, G. Endrödi, S. Katz, and A. Schäfer, The QCD equation of state in background magnetic fields, J. High Energy Phys. 08 (2014) 177.

[31] C. Bonati, M. D’Elia, M. Mariti, M. Mesiti, F. Negro, A. Rucci, and F. Sanfilippo, Magnetic field effects on the static quark potential at zero and finite temperature, Phys. Rev. D 94, 094007 (2016).

[32] K.-I. Kondo, S. Kato, A. Shibata, and T. Shinohara, Quark confinement: Dual superconductor picture based on a nonAbelian Stokes theorem and reformulations of Yang-Mills theory, Phys. Rep. 579, 1 (2015).

[33] S. N. Nedelko and V. E. Voronin, Finite size effects in the free energy density for Abelian (anti-)self-dual gluon field in $S U$ (3) gluodynamics, Phys. Part. Nucl. Lett. 16, 553 (2019).

[34] L. D. Faddeev, Mass in quantum Yang-Mills theory: Comment on a Clay millenium problem, arXiv:0911.1013.

[35] L. D. Faddeev, A couple of methodological comments on the quantum Yang-Mills theory, Theor. Math. Phys. 181, 1638 (2014).

[36] B. V. Galilo and S. N. Nedelko, Impact of the strong electromagnetic field on the QCD effective potential for homogeneous Abelian gluon field configurations, Phys. Rev. D 84, 094017 (2011).

[37] L. D. Faddeev, Notes on divergences and dimensional transmutation in Yang-Mills theory, Teor. Mat. Fiz. 148, 133 (2006) [Theor. Math. Phys. 148, 986 (2006)].

[38] S. V. Shabanov and J. R. Klauder, Towards a nonperturbative path integral in gauge theories, Phys. Lett. B 456, 38 (1999).

[39] S. V. Shabanov, Geometry of the physical phase space in quantum gauge systems, Phys. Rep. 326, 1 (2000).

[40] Y. Cho, Colored Monopoles, Phys. Rev. Lett. 44, 1115 (1980); Erratum, Phys. Rev. Lett. 44, 1566 (1980). 
[41] L. D. Faddeev and A. J. Niemi, Spin-charge separation, conformal covariance and the SU(2) Yang-Mills theory, Nucl. Phys. B776, 38 (2007).

[42] K. I. Kondo, T. Murakami, and T. Shinohara, Yang-Mills theory constructed from Cho-Faddeev-Niemi decomposition, Prog. Theor. Phys. 115, 201 (2006).

[43] L. D. Faddeev, Separation of scattering and selfaction revisited, arXiv:1003.4854.

[44] T. Vachaspati, Kinks and Domain Walls (Cambridge University Press, Cambridge, England, 2006).

[45] Y. M. Cho, D. G. Pak, P. M. Zhang, and L. P. Zou, Weyl symmetric structure of qcd vacuum, Phys. Rev. D 86, 045025 (2012).

[46] M. Bordag, E. Elizalde, and K. Kirsten, Heat kernel coefficients of the Laplace operator on the D-dimensional ball, J. Math. Phys. (N.Y.) 37, 895 (1996).

[47] K. Kirsten, Spectral Functions in Mathematics and Physics (Chapman and Hall/CRC, London, 2001).

[48] S. Deser, L. Griguolo, and D. Seminara, Gauge Invariance, Finite Temperature and Parity Anomaly in $D=3$, Phys. Rev. Lett. 79, 1976 (1997).

[49] C. Wiesendanger and A. Wipf, Running coupling constants from finite size effects, Ann. Phys. (N.Y.) 233, 125 (1994).

[50] G. Cognola, K. Kirsten, and S. Zerbini, One loop effective potential on hyperbolic manifolds, Phys. Rev. D 48, 790 (1993).

[51] V. Braguta, A. Kotov, D. Kuznedelev, and A. Roenko, Study of the confinement/deconfinement phase transition in rotating lattice $\mathrm{SU}(3)$ gluodynamics, JETP Lett. 112, 6 (2020).

[52] A. Yamamoto and Y. Hirono, Lattice QCD in Rotating Frames, Phys. Rev. Lett. 111, 081601 (2013).

[53] A. Pais, Spherical spinors in a Euclidean 4-space, Proc. Natl. Acad. Sci. U.S.A. 40, 835 (1954).

[54] B. Carter and R. G. McLenaghan, Generalized total angular momentum operator for the Dirac equation in curved spacetime, Phys. Rev. D 19, 1093 (1979).

[55] V. N. Shapovalov, Symmetry and classification of the Dirac-Fock equation, Teor. Mat. Fiz. 197, 208 (2018) [Theor. Math. Phys. 197, 1572 (2018)].

[56] A. I. Breev and A. V. Shapovalov, The Dirac equation in an external electromagnetic field: Symmetry algebra and exact integration, J. Phys. Conf. Ser. 670, 012015 (2016).

[57] M. Abramowitz and I. A. Stegun, Handbook of Mathematical Functions with Formulas, Graphs, and Mathematical Tables (U.S. Department of Commerce, National Bureau of Standards, Washington, D.C., 1972).

[58] F. W. J. Olver, Asymptotics and Special Functions, 1st ed. (A K Peters/CRC Press, New York, 1997), Chapter Differential Equations with a Parameter: Expansions in Elementary Functions, pp. 382-386.

[59] DLMF, NIST Digital Library of Mathematical Functions, edited by F. W. J. Olver, A. B. O. Daalhuis, D. W. Lozier, B. I. Schneider, R. F. Boisvert, C. W. Clark, B. R. Miller, and B. V. Saunders, http://dlmf.nist.gov/, Release 1.0.23 of 2019-06-15. 